\title{
Physical parameters of rich LMC clusters from modeling of deep HST colour-magnitude diagrams
}

\author{
L. O. Kerber and B. X. Santiago
}

\author{
Universidade Federal do Rio Grande do Sul, IF, CP 15051, Porto Alegre 91501-970, RS, Brazil \\ e-mail: kerber@if.ufrgs.br
}

Received 30 October 2004 / Accepted 12 December 2004

\begin{abstract}
We present the analysis of deep colour-magnitude diagrams (CMDs) of five rich LMC clusters. The data were obtained with HST/WFPC2 in the $F 555 W(\sim V)$ and $F 814 W(\sim I)$ filters, reaching $V_{555} \sim 25$. The sample of clusters is composed of NGC 1805 and NGC 1818, the youngest ones $(\tau<100 \mathrm{Myr})$, NGC 1831 and NGC 1868, of intermediate-age $(400<\tau<$ $1000 \mathrm{Myr}$ ), and Hodge 14, the oldest ( $\tau>1200 \mathrm{Myr}$ ). We discuss and apply a statistical method for correcting the CMD for sampling incompleteness and field star contamination. Efficient use of the CMD data was made by means of direct comparisons of the observed to model CMDs. The CMD modeling process generates a synthetic Main Sequence (MS), where we introduce as model inputs the information about age, chemical composition, present day mass function (PDMF), fraction of unresolved binaries, distance modulus and light extinction. The photometric uncertainties were empirically determined from the data and incorporated into the model as well. Statistical techniques of CMD comparisons using 1 and 2 dimensions are presented and applied as an objective method to assess the compatibility between model and data CMDs. By modeling the CMDs from the central region we infer the metallicity $(Z)$, the intrinsic distance modulus $\left((m-M)_{0}\right)$ and the reddening value $(E(B-V))$ for each cluster. We also determined the age for the clusters with $\tau>400$ Myr. By means of two-dimensional CMD comparisons we infer the following values: for NGC 1805, $Z=0.007 \pm 0.003,(m-M)_{0}=18.50 \pm 0.11, E(B-V)=0.03 \pm 0.01$; for NGC 1818, $Z=0.005 \pm 0.002,(m-M)_{0}=18.49 \pm 0.14, E(B-V) \sim 0.00 ;$ for NGC 1831, $Z=0.012 \pm 0.002, \log (\tau / \mathrm{yr})=8.70 \pm 0.03$, $(m-M)_{0}=18.70 \pm 0.03, E(B-V) \sim 0.00$; for NGC 1868, $Z=0.008 \pm 0.002, \log (\tau / \mathrm{yr})=8.95 \pm 0.03,(m-M)_{0}=18.70 \pm 0.03$, $E(B-V) \sim 0.00$; for Hodge 14, $Z=0.008 \pm 0.004, \log (\tau / \mathrm{yr})=9.23 \pm 0.10,(m-M)_{0}=18.51 \pm 0.13, E(B-V)=0.02 \pm 0.02$. Taking into account the uncertainties, these values are in accordance with the ones obtained applying the one-dimensional CMD analysis, adding reliability to these determinations.
\end{abstract}

Key words. galaxies: star clusters - Magellanic Clouds - Hertzsprung-Russell (HR) and C-M diagrams

\section{Introduction}

The excellent spatial resolution of the Hubble Space Telescope (HST) has made it possible to study in detail the stellar content of nearby galaxies, even in dense environments such as their central regions or inside rich stellar clusters (Hunter et al. 1996, 1997; Elson et al. 1997; Sarajedini et al. 1998; Gallart et al. 1999; Keller et al. 2000). The Large Magellanic Cloud (LMC) is a unique nearby case of a gas rich, star forming, irregular galaxy containing thousands of clusters with varying masses, ages and metallicities (Westerlund 1990; Olszewski et al. 1996). This diverse cluster system is quite different from that of our own Galaxy, where we find a dichotomy between relatively sparse and young open disk clusters on one side, and rich and old globular halo clusters on the other. Therefore, the LMC cluster system is a useful record of the history of star formation, chemical enrichment and dynamics of a quite distinct type of galaxy (Bica et al. 1998; Olsen et al. 1998). In particular, as examples of both old and young rich LMC clusters exist, the correct characterization of their internal properties is useful to study several effects related to gravitational dynamics of $\mathrm{N}$-body systems, including mass segregation and stellar evaporation (Santiago et al. 2001; de Grijs et al. 2002a). These effects in turn are an important step towards recovering the cluster initial conditions and assessing the universality of the initial mass function (Goodwin 1997; Paresce \& de Marchi 2000; Kroupa et al. 2001; Kroupa 2002; de Grijs et al. 2002c).

The HST-based colour-magnitude diagrams (CMDs) of LMC clusters often reach down to magnitudes well below their main-sequence turn-off, allowing age and metallicity estimates through isochrone fitting and derivation of luminosity and mass functions through star counts. Determination of a cluster's physical parameters, however, is hampered by several effects such as extinction by intervening dust, CMD contamination by field stars, sample incompleteness, and the presence of unresolved binaries. As a result, isochrone fitting, the most often used means to extract useful parameters from CMD data, suffers from a degeneracy in the possible solutions found. Often 
little can be done to avoid these ambiguous solutions, since by varying age, metallicity, distance modulus and extinction values within reasonable limits, different isochrones can be equally successful in fitting a given cluster main-sequence, its turn-off and the giant branch. For rich clusters, however, given the dense sampling of stars and the relative ease with which field contamination can be compensated for, alternative methods of inferring physical and structural parameters may help raise this degeneracy and yield unique solutions for these parameters.

We have been working on methods to extract physical parameters from an observed CMD by directly comparing it to model CMDs. This type of approach has also been developed by other authors (Hernandez et al. 1999, 2000; Kerber et al. 2001; Carrera et al. 2002; Dolphin 2002). In the case of cluster CMDs, which represent a single stellar population, the artificial model CMDs are generated according to a choice of age, metallicity, distance, extinction, mass function and binary fraction. Our method relies on the use of more than one objective statistic that quantify how similar the observed and model CMDs being compared are. Therefore, it allows the determination of a range of the aforementioned parameters that result in reliable solutions. A preliminary application of such techniques has been carried out by Kerber et al. (2002) for the rich LMC cluster NGC 1831. This cluster is one among a sample of 7 rich clusters that has been observed by several HST instruments as part of a cycle 7 project entitled "Formation and evolution of rich LMC clusters" (Beaulieu et al. 1999; Elson et al. 1999; Johnson et al. 2001; Castro et al. 2001; de Grijs et al. 2002c). In this paper we extend the determination of the same parameters for 5 of the clusters in the sample, making use of improved statistical methods. We concentrate here on ages, metallicities, extinction values and distance moduli. In an upcoming paper, the present day mass function (PDMF), as well as its dependence on position within the clusters, will be addressed. The separate analysis of the PDMF is due to the different effect it has on the distribution of stars on the CMD plane: while the other parameters determine the position of the several populated loci on a CMD, the mass function reflects how stars are spread along these loci. Furthermore, the PDMF varies with position inside the cluster, while the other 4 parameters mentioned here globally apply to it.

The 5 clusters studied here are listed in Table 1, along with their main properties as found in the literature.

The current paper is divided as follows. In Sect. 2 we present our photometric data and the way we have dealt with the several observational effects and biases that are common to them. These include sample photometric uniformization, field decontamination and correction for sample incompleteness. In Sect. 3 we describe our algorithm for generating model CMDs that mimic the observational HST data. Section 4 presents the statistical methods of CMD comparison adopted, as well as their application to control experiments to test their applicability. In Sect. 5 we apply our CMD modeling and comparison techniques to the observed LMC cluster CMDs, extracting reliable values for their global parameters. Finally, we present a summary of our results and our conclusions in Sect. 6.
Table 1. Cluster parameters as found in the literature. Column 1: cluster name; Col. 2: angular distance to LMC $\left(\theta_{\mathrm{LMC}}\right)$; Col. 3: central density $\left(\rho_{0}\right)$; Col. 4 : half-light radius $\left(R_{\mathrm{hl}}\right)$; Col. 5: core radius $\left(R_{\text {core }}\right)$; Col. 6: range of $Z\left(\approx 0.019 \times 10^{[\mathrm{Fe} / \mathrm{H}]}\right)$; Col. 7: range of $\log (\tau / \mathrm{yr})$.

\begin{tabular}{lcccccl}
\hline \hline Cluster & $\begin{array}{c}\theta_{\mathrm{LMC}}^{a} \\
\left({ }^{\circ}\right)\end{array}$ & $\begin{array}{c}\rho_{0}^{a} \\
\left(\mathrm{stars} /{ }^{\prime \prime 2}\right)\end{array}$ & $\begin{array}{c}R_{\mathrm{hl}}^{a} \\
\left(^{\prime \prime}\right)\end{array}$ & $\begin{array}{c}R_{\text {core }}^{b} \\
\left({ }^{\prime \prime}\right)\end{array}$ & $10^{2} Z$ & $\log (\tau / \mathrm{yr})$ \\
\hline NGC 1805 & 4.1 & 1.88 & 7.2 & 5.5 & $0.8-1.9^{c}$ & $6.90-7.30^{d}$ \\
NGC 1818 & 3.8 & 2.25 & 10.4 & 10.1 & $0.2-1.9^{c, e}$ & $7.30-7.70^{d}$ \\
NGC 1831 & 5.1 & 3.06 & 14.4 & 18.3 & $0.5-1.9^{f, g}$ & $8.40-8.80^{h, i}$ \\
NGC 1868 & 5.9 & 3.38 & 7.2 & 6.7 & $0.1-0.6^{g, j}$ & $8.70-9.00^{g, k}$ \\
Hodge 14 & 3.9 & 0.75 & 11.6 & 7.4 & $0.2-0.6^{g, l}$ & $9.15-9.40^{g, m}$ \\
\hline
\end{tabular}

Reference list: ${ }^{a}$ Santiago et al. (2001); ${ }^{b}$ Mackey \& Gilmore (2003);

${ }^{c}$ Johnson et al. (2001); ${ }^{d}$ de Grijs et al. (2002b); ${ }^{e}$ Meliani et al. (1994);

${ }^{f}$ Chiosi et al. (1989); ${ }^{g}$ Olszewski et al. (1991); ${ }^{h}$ Meurer et al. (1990);

${ }^{i}$ Kerber et al. (2002); ${ }^{j}$ Chiosi et al. (1986); ${ }^{k}$ Girardi et al. (1995);

${ }^{l}$ Jensen et al. (1988); ${ }^{m}$ Bomans et al. (1995).

\section{The data}

We use data taken with the Wide Field and Planetary Camera (WFPC2) on board of HST for 5 rich LMC clusters and nearby fields. These data are part of the G07307 project entitled "Formation and Evolution of Rich Star Clusters in the LMC". For each cluster, images were obtained using the $F 555 W(\sim V)$ and $F 184 W(\sim I)$ filters. Most of the photometry had already been performed: cluster stellar luminosity functions (LFs) were built and analysed by Santiago et al. (2001), de Grijs et al. (2002a,b,c); field stellar populations were studied by Castro et al. (2001) and Javiel et al. (2005). Field coordinates, image reduction and photometry processes are described in detail by those authors.

To distinguish the different fields, we call "on-cluster" those that are in a cluster's direction and "off-cluster" those located in a nearby field position. The "on-cluster" field is a composition of the CEN and HALF fields, as defined by Santiago et al. (2001). These have the higher-resolution Planetary Camera (PC) centered on the cluster's center and half-light radius, respectively. In Fig. 1 we show the on-cluster field of NGC 1868 projected on the sky. The CCD borders are also drawn. The CEN/HALF arrangement provides the following advantages: 1 ) the best WFPC2 spatial resolution $\left(0.046^{\prime \prime} /\right.$ pix $)$ available in the dense cluster center; 2$)$ a large solid angle coverage, thus increasing the stellar sample; 3) empirical determination of photometric uncertainties, since the overlap region contains stars with two independent photometric measurements (Sect. 2.3).

The "off-cluster" field is located at about 7.3 arcmin away form the cluster center. This field is used for statistical decontamination of the "on-cluster" sample of field stars.

Table 2 presents, for each field and filter, the total exposure time and conservative estimates of saturation and detection magnitude limits. The saturation limit was obtained directly from photometry and the detection limit was given by the typical magnitude at which the completeness falls to less than $50 \%$ (see Sect. 2.4.1). 
Table 2. General characteristics of on-cluster (CEN and HALF) and off cluster fields. Column 1 lists the field name. The number of exposures are shown in Cols. 2 and 3 for the $I$ and $V$ bands, respectively. The total exposure times are presented in Cols. 4 and 5. Columns 6 and 7 show the corresponding saturation limits. Finally, the two last columns present the detection limits.

\begin{tabular}{lcccccccc}
\hline \hline Field/image & $N_{I}$ & $N_{V}$ & $t_{I}(s)$ & $t_{V}(s)$ & $I_{\text {sat }}$ & $V_{\text {sat }}$ & $I_{\text {det }}$ & $V_{\text {det }}$ \\
\hline on/CEN & 3 & 3 & 900 & 420 & 17.0 & 18.0 & 22.5 & 23.0 \\
on/HALF & 3 & 3 & 2500 & 2500 & 18.5 & 19.5 & 23.5 & 24.0 \\
off & 2 & 2 & 800 & 1200 & 17.5 & 18.5 & 24.0 & 24.5 \\
\hline
\end{tabular}

\subsection{The on-cluster composition}

As just mentioned, the final on-cluster sample was made by the combination of the CEN and HALF samples. In order to make them compatible we applied geometric and photometric cuts and an empiric method to place the photometry of both samples on a uniform scale.

\subsubsection{Geometric and photometric cuts}

To avoid a double sampling of stars located in the overlap region between the CEN and HALF fields, we applied geometrical cuts selecting sequentially the following regions: 1) $\mathrm{PC} / \mathrm{CEN}$ (priorizing high resolution in the cluster center); 2) WFPC2/HALF, except within the region covered by the $\mathrm{PC} / \mathrm{CEN}$ (priorizing the deeper field in the outer cluster regions); 3) WFCs/CEN that were not imaged by the HALF field (using the maximum available information).

Simultaneously to the geometrical sampling, we also cutoff stars brighter the saturation limit of each field, listed in Table 2. The faint magnitude limits were imposed at a later stage and are discussed in Sect. 2.4.1.

\subsubsection{Photometric uniformization}

The photometry on our on-cluster data presents some systematic biases as a function of exposure time (CEN vs. HALF) and chip (WFCs vs. PC). Several authors have detected this kind of effect in WFPC2 data (Kerber et al. 2002; de Grijs et al. 2002a; Johnson et al. 2001; Casertano \& Muchtler 1998; Brocato et al. 2001). The amplitude of the effect is not negligible and may reach $\sim 0.05$ in magnitude and $\sim 0.10$ in colour. The possible origins of these biases are the Charge Transfer Effect (CTE), variable aperture corrections or zero points (Johnson et al. 1999); there is not a consensus as to the method of correction or its amplitude.

Our method of photometric uniformization was to empirically measure such magnitude and colour changes and to apply appropriated shifts to the data when necessary. We first chose the WFCs/HALF data as a reference. This choice is based on the observed uniformity among three WFC chips and the longer exposure time (Table 2) and lower field density of the HALF field.

To quantify the exposure time effect we used the independent photometric measurements of stars in common

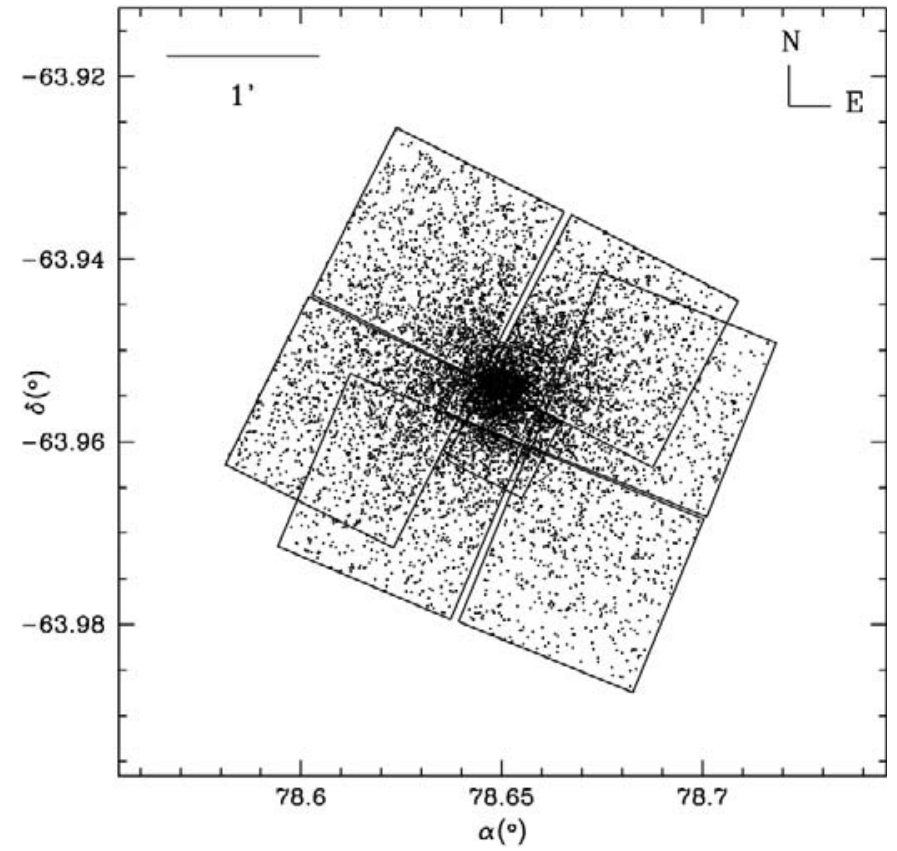

Fig. 1. The "on-cluster" field of NGC 1868. The CEN field has the PC centered in the cluster center and the HALF field has the PC centered in the half-light radius.

between the CEN and HALF fields (Fig. 1). In Fig. 2 we show $I_{814}(\mathrm{HALF})-I_{814}(\mathrm{CEN})\left(V_{555}(\mathrm{HALF})-V_{555}(\mathrm{CEN})\right)$ as a function of $I_{814}(\mathrm{CEN})\left(V_{555}\right)$ in the upper (lower) panel, for NGC 1831. Except for the bright end, where saturation affects first the deeper HALF field, the systematic (HALF - CEN) magnitude differences are almost constant $(\sim 0.1 \mathrm{mag})$. The data are very well fitted by the nearly horizontal lines shown, with residuals of $\simeq 0.05$. As the exposure time effect has the same sign in both filters, the systematic effect on $(V-I)$ colour is small, $\sim 0.04$, in the sense that the WFCs/CEN data are found to be redder than the WFCs/HALF data. The WFCs photometry was made uniform by mapping the WFCs/CEN magnitudes onto the WFCs/HALF data using the linear fits just described.

The PC/CEN and PC/HALF data were also brought to the same scale as the WFCs/HALF. However, the number of stars in common between PC/HALF and WFCs/CEN is small. The $\mathrm{PC} / \mathrm{CEN}$ and WFCs/HALF comparison turned out to be of low quality, since the latter magnitudes are unreliable (the central cluster regions are too cluttered for the lower-resolution WFC chips). Therefore, this chip-dependent uniformization could not be made by using direct magnitude comparisons for the same stars. Systematic photometric corrections to the PC data were made with the aid of fiducial lines of MS stars, following the procedure of Kerber et al. (2002). In brief, the MS fiducial line is defined by the median $V_{555}-V_{814}$ colour at different magnitude bins along the MS. By comparing the fiducial lines described by PC stars with those of WFCs stars, we found that the former were systematically bluer in all clusters, the amplitude of the effect being in the range 0.01-0.08 mag. This systematic effect applies to both CEN and HALF images. Once corrected for the exposure time effect, the WFCs fiducial lines are very stable, always occupying loci in the CMD very 

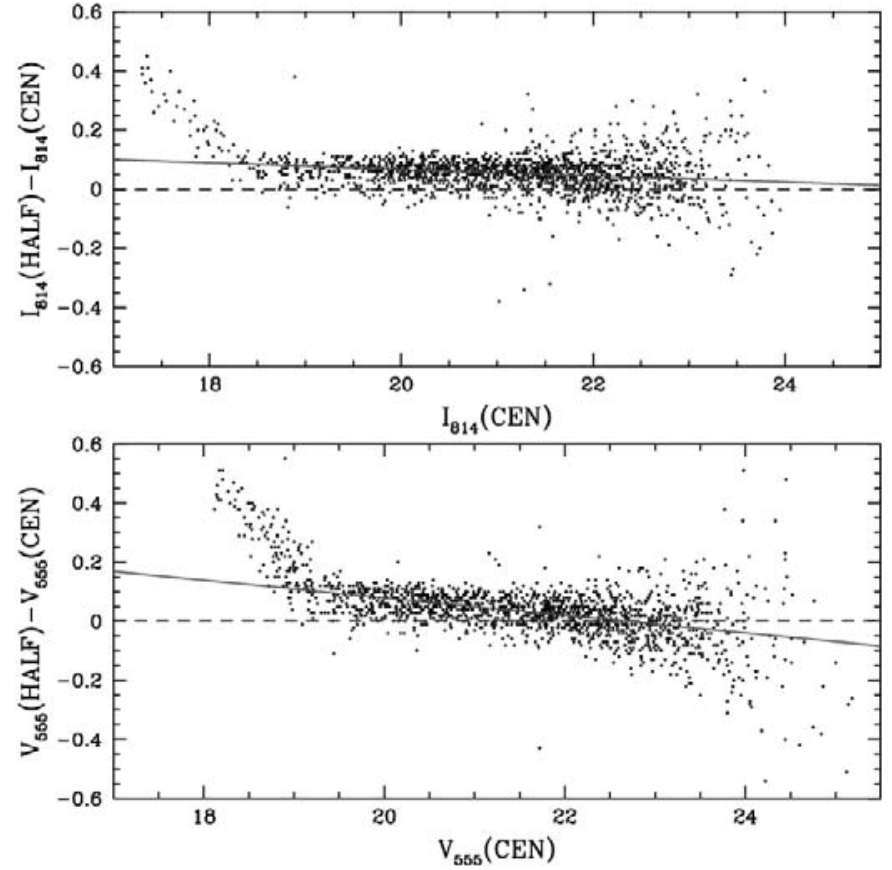

Fig. 2. The exposure time effect shown by photometric comparison between WFCs/CEN and WFCs/HALF common data for NGC 1831. The upper (lower) panel shows this comparison for the $F 814 \mathrm{~W}$ $(F 555 W)$ filter, where the solid line is a linear fit.

close to each other. Thus, we transposed the PC fiducial lines to the WFC locus by means of the mean colour difference between their fiducial lines. In practice, this was done by changing the PC I band magnitudes accordingly.

We have also searched for systematic effects in the offcluster data, but none was found.

\subsection{The observed CMDs}

In Figs. 3 and 4 we present, for all 5 clusters in our sample, the final on-cluster and off-cluster CMDs, respectively. The sequence of panels $(\mathrm{a}-\mathrm{e})$ in Fig. 3 roughly corresponds to an age sequence, as can be seen by the cluster MS termination point. Unfortunately, for NGC 1805 and NGC 1818 clusters, the MS stretches all the way up to the saturation limit of our data $\left(V_{555}=18.0\right)$. Consequently, we are not able to determine the ages of these systems from our CMD analysis.

Comparing each panel of Fig. 3 with its corresponding one in Fig. 4, we can easily detect the field star contamination in the on-cluster data by the presence of a subgiant and red giant branch $\left(V_{555} \sim 22\right)$, made up of stars of varying ages. Also, there is a clear nonuniformity in the off-cluster CMDs, revealing the different field populations in the LMC. In comparison with the others fields, the fields associated with the younger clusters, which are close to the LMC bar, are richer and more contaminated by younger stars. Castro et al. (2001) and more recently Javiel et al. (2005) analysed these off-cluster CMDs determining the different Star Formation Histories (SFHs) of these fields.

Together with field star contamination, the on-cluster CMDs suffer from another important selection effect: sample

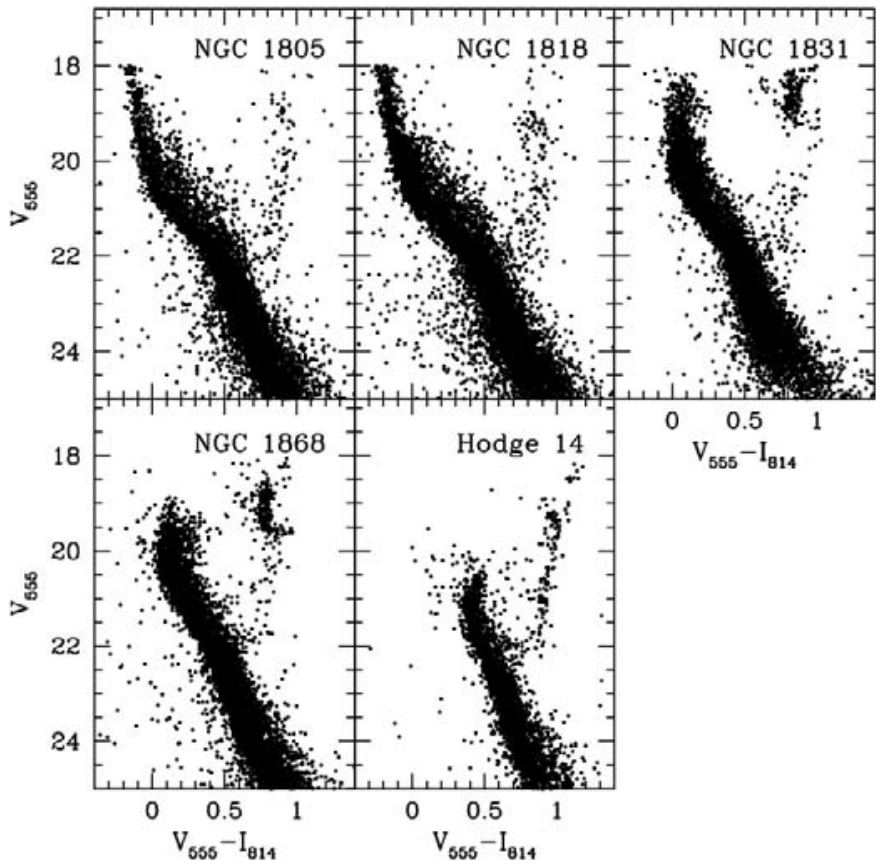

Fig. 3. The on-cluster CMDs for all clusters in our sample.

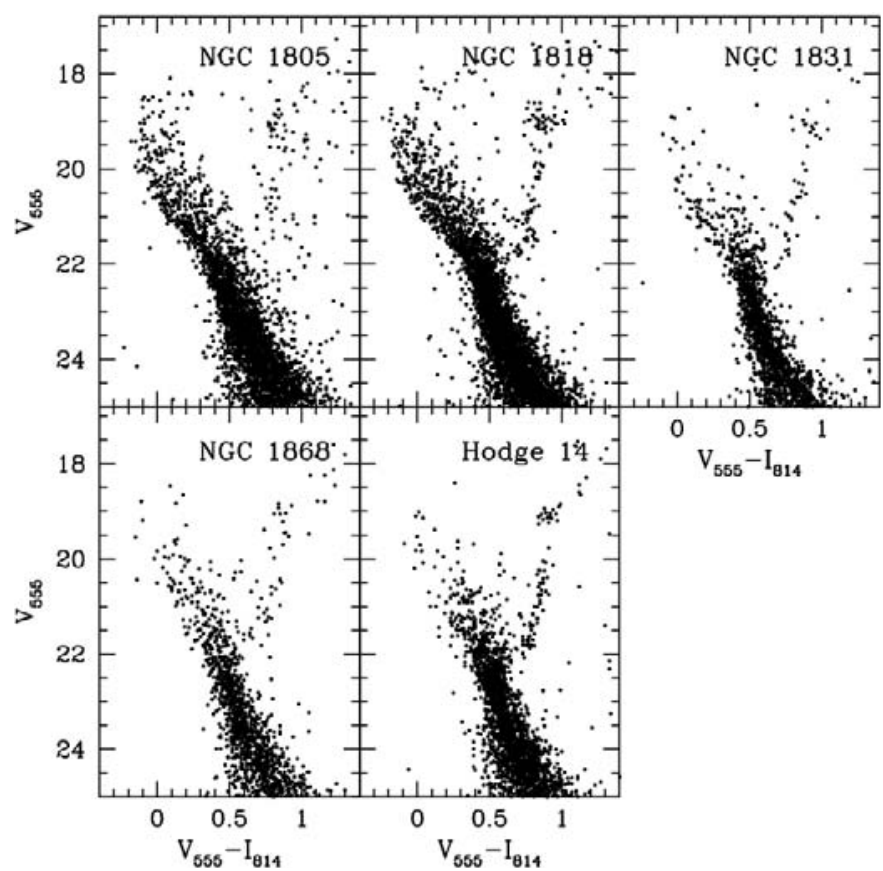

Fig. 4. The off-cluster CMDs for all clusters in our sample.

incompleteness. Our CMD modeling algorithm does not incorporate such effects. Therefore it is crucial to correct for both effects on the observed CMDs in order to place models and data on an equal footing. This is done in Sect. 2.4. However, as a necessary first step, we needed to quantify the random photometric uncertainties. These are the major cause of the spreading of stars on the CMD plane, and, as such, will also be very useful for the CMD modeling algorithm. 
Table 3. The random photometric uncertainties estimated for CEN and HALF stars in the $F 814 W(\sim I)(\mathrm{Col} .2)$ and $F 555 W(\sim V)(\mathrm{Col} .3)$ filters. They are shown as a function of the empirically determined dispersion among independent photometric measurements.

\begin{tabular}{ccc}
\hline \hline & $F 814 W(\sim I)$ & $F 555 W(\sim V)$ \\
\hline$\sigma_{\text {cen }}$ & $0.86 \sigma_{\mathrm{obs}, I}$ & $0.93 \sigma_{\mathrm{obs}, V}$ \\
$\sigma_{\text {half }}$ & $0.51 \sigma_{\mathrm{obs}, I}$ & $0.38 \sigma_{\mathrm{obs}, V}$ \\
\hline
\end{tabular}

\subsection{Random photometric uncertainties}

The random photometric uncertainties, together with the unresolved binarism, are the main source of spread in our HST/WFPC2 CMDs. Therefore, a suitable assessment of these uncertainties in both filters is fundamental to correctly incorporate this effect into CMD modeling process.

Due to the overlap region imaged by both HALF and CEN fields (Fig. 1), we have a stellar sample with two independent photometric measurements in the on-cluster field. By comparing these measurements we were able to quantify the random photometric uncertainties of on-cluster stars in both filters. As commented earlier (Sect. 2.1.2), the only reliable sample of stars in common is that made of WFCs/CEN vs. WFCs/HALF data. Thus, we binned the data in magnitude for each filter and computed the dispersion, $\sigma_{\mathrm{obs}}$, around the linear fit solutions shown in Fig. 2. $\sigma_{\mathrm{obs}}$ is the composition of two independent realizations. We thus have

$\sigma_{\mathrm{obs}}^{2}=\sigma_{\mathrm{cen}}^{2}+\sigma_{\text {half }}^{2}$,

where $\sigma_{\text {cen }}$ and $\sigma_{\text {half }}$ are the CEN and HALF dispersions. This equation applies to both filters.

In a more simplistic approach, Kerber et al. (2002) assumed that the CEN and HALF uncertainties were equal, disregarding the difference in exposure time. In this work we improved this error treatment by assuming that the uncertainty is proportional to the inverse of the square root of the exposure time $(t)$. Thus, we can write

$\frac{\sigma_{\text {cen }}}{\sigma_{\text {half }}}=\frac{\sqrt{t_{\text {half }}}}{\sqrt{t_{\text {cen }}}}$

which yields the following expressions

$\sigma_{\text {cen }}=\sigma_{\text {obs }}\left(\frac{t_{\text {half }}}{t_{\text {cen }}+t_{\text {half }}}\right)^{1 / 2}$

$\sigma_{\text {half }}=\sigma_{\text {obs }}\left(\frac{t_{\text {cen }}}{t_{\text {cen }}+t_{\text {half }}}\right)^{1 / 2}$.

Using the total exposure times (Table 2) in the above expression we estimated the values of $\sigma_{\text {cen }}$ and $\sigma_{\text {half }}$ in both filters. They are presented in Table 3 as a function of $\sigma_{\text {obs }}$. As expected, $\sigma_{\text {cen }}$ is significantly larger than $\sigma_{\text {half }}$, since the CEN field is shallower. Figure 5 shows a typical dependence of the $\sigma_{\text {obs }}$, $\sigma_{\text {cen }}$ and $\sigma_{\text {half }}$ as a function of magnitude. The upper panel shows the results for the $F 814 W(\sim I)$ filter, the middle panel gives plots $F 555 W(\sim V)$ dispersions, and the lower panels shows the colour uncertainties.
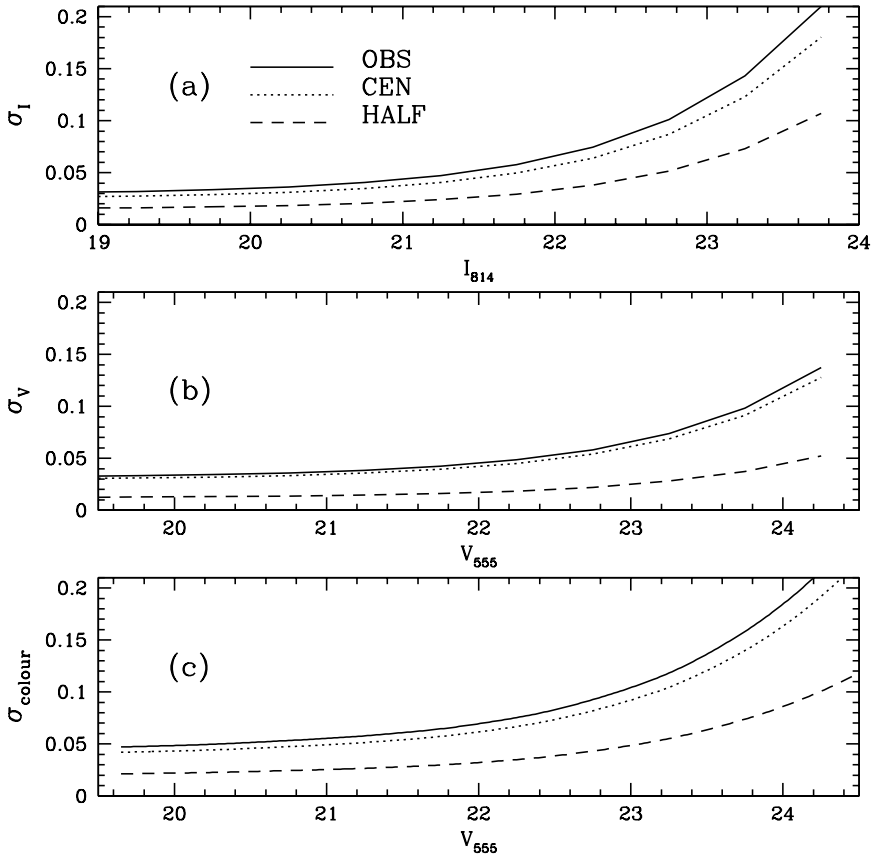

Fig. 5. A typical empirical determination of photometric uncertainties (for NGC 1818) as a function of magnitude. The solid line presents the uncertainty $\left(\sigma_{\mathrm{obs}}\right)$ obtained directly by independent measurements. The dotted and dashed lines correspond to $\sigma_{\text {cen }}$ and $\sigma_{\text {half }}$, respectively. The uncertainties for $F 814 \mathrm{~W}$ and $F 555 \mathrm{~W}$ are shown in the panels a) and b), respectively. Panel c) presents the $\sigma_{\text {colour }}$ determination for the MS stars.

The colour dispersion is determined from those in the $F 555 \mathrm{~W}$ and $F 814 \mathrm{~W}$ magnitudes. Since the magnitudes in the two filters are independent, the colour uncertainty $\left(\sigma_{\text {colour }}\right)$ was given by quadratic sum of the filter uncertainties:

$\sigma_{\text {colour }}^{2}=\sigma_{V}^{2}+\sigma_{I}^{2}$.

Analysing Fig. 5 and Table 3 one can notice that typically $\sigma_{\text {cen,colour }} \sim 2 \sigma_{\text {half,colour }}$, yielding a more realistic error treatment than the one used by Kerber et al. (2002).

As commented in Kerber et al. (2002), we did not have two independent and overlapping WFPC2 off-cluster images. Therefore, we could not apply the same method described above to quantify the photometric uncertainties of field stars. The solution found was to employ the same estimate as for the CEN on-cluster stars. As the off-cluster field is deeper and sparser then the on-cluster ones, we can expected that this approximation yields to an overestimate of the photometric uncertainties in the off-cluster data. Fortunately, these stars are used only for statistical subtraction of field contamination from the on-cluster CMD, and we observe that the decontamination result is fairly independent of adopted photometric uncertainties (Kerber et al. 2002).

\subsection{Selection effects}

\subsubsection{Sample incompleteness}

Sample incompleteness is due to the difficulties involved in the detection and classification of sources at faint magnitude limits. 
It is caused by two main factors: overlapping of stellar profiles due to crowding and background noise. Therefore, in a cluster the incompleteness will depend not only on apparent magnitude, but also on spatial position. It means that faint stars in dense stellar regions are the ones that suffer most from this effect. Santiago et al. (2001) previously discussed and measured the completeness for the on-cluster data. These authors carried out experiments where artificial stars were added to the cluster image and subjected to the same sample selection as the real stars. The completeness $c$ of each real star was estimated as the fraction of artificial stars of similar magnitude and location which were successfully recovered in the experiments. Therefore, they assigned for each star a weight $w$ due to completeness given by the inverse of its value $(w=1 / c)$. This weight corresponds to the total number of stars similar to the observed one which should be detected and measured in an ideal image.

Since completeness is a function of position within the cluster, incorporating this effect into our model CMDs would require modeling the distribution of artificial stars in the cluster. We bypass this issue by compensating the observed CMD, where stellar positions relative to cluster center are already known, for incompleteness effects. In each on-cluster CMD this correction for sample incompleteness was made through the following steps:

(1) we arbitrarily defined the cluster MS region in the CMD by means of guiding straight lines;

(2) we defined a MS fiducial line taking the median colour at different magnitude bins. This line establishes a univocal relation between the magnitude $V_{555}$ and colour $V_{555}-I_{814}$ along the MS;

(3) given the $V_{555}$ magnitude of the $i$ th observed star, we found the corresponding $V_{555}-I_{814}$ colour in the fiducial line;

(4) we spread out $w_{i}-1$ extra stars from this CMD position using the measured photometric uncertainties. We assume a Gaussian distribution of uncertainties both in $V_{555}$ and $V_{814}$;

(5) we repeated this procedure from step (3) for all observed stars, completing the CMD down to $V_{555}=24.5$. However, the CMD analysis was restricted to magnitude brighter limits, typically of $V_{555} \simeq 23.5$, where incompleteness is not significantly less than $50 \%$.

At the end of this process we obtain a CMD with a complete sample of stars both in number and in position. Notice that we used the observational constraints given by the fiducial line of the MS, the completeness weight and the empirically determined photometric uncertainties.

Figure 6 depicts, for NGC 1868, the method for sample incompleteness correction in the CMDs. Panel (a) shows the region arbitrarily defined as the MS and the MS fiducial line. The empirically estimated $1-\sigma$ colour spread is shown in panel (b). Panel (c) presents the extra stars from incompleteness correction added to the observed ones. Finally, the "complete" oncluster CMD, cut at $V_{555}=24.5$, is presented in panel (d).

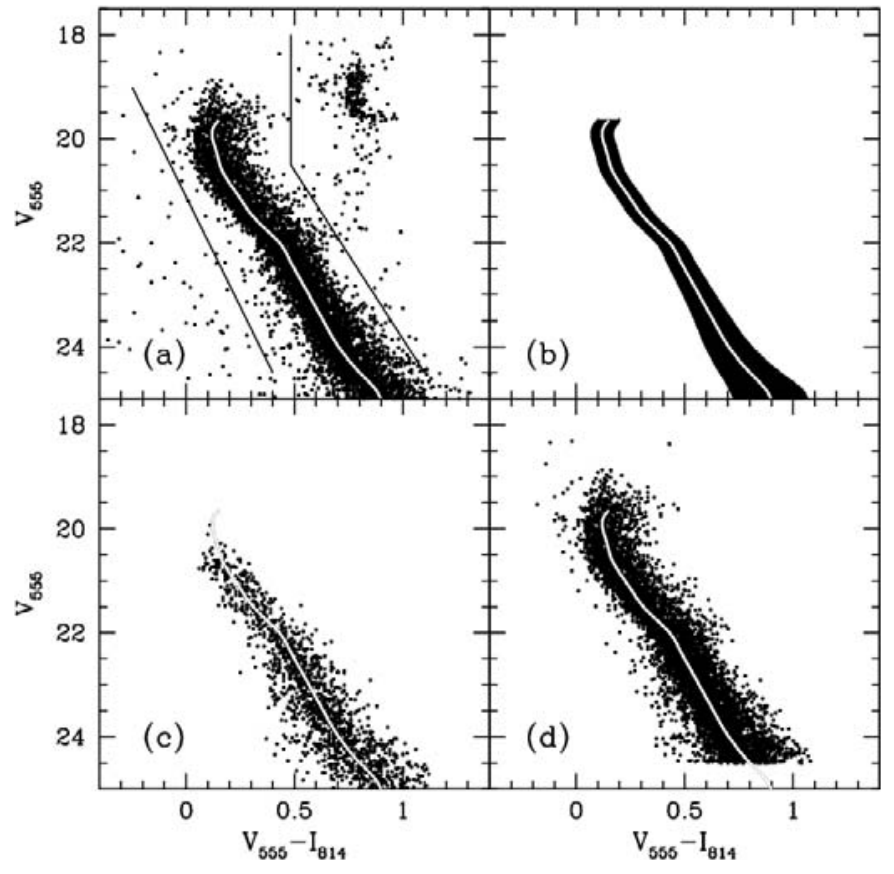

Fig. 6. Correcting the NGC 1868 on-cluster CMD for incompleteness: a) the observed CMD, the region defined as MS (inside the guiding solid lines) and the fiducial MS line (white line); b) $1-\sigma_{\text {colour }}$ spread from the fiducial line; $\mathbf{c}$ ) the extra stars from sample incompleteness correction; d) the complete CMD.

\subsubsection{Field star subtraction}

The first step in field star subtraction from the on-cluster CMD was made in the correction for the sample incompleteness, where we exclude all stars outside the defined cluster MS region in the CMD. We therefore eliminated all evolved stars, as well as objects which are likely to be foreground stars or remaining non-stellar sources in the sample (distant galaxies, spurious image features, etc.).

The second step was a statistical removal of field stars located along the cluster MS. We use here the same method proposed by Kerber et al. (2002). In brief, this method atributes a probability of each on-cluster star being a field star and, according to this probability, randomly removes stars from the on-cluster CMD. The probability calculation takes into account CMD positions, spatial position within the cluster and photometric uncertainties. The method is based on the hypothesis that the positions of the off-cluster stars in the CMD represent the most likely positions for field stars on any similar CMD. Therefore, comparing on-cluster CMDs from concentric regions in each cluster to the off-cluster CMD, and taking into account corresponding the solid angles, we determine a statistical cluster CMD. We refer the reader to Kerber et al. (2002) for details.

Figure 7 displays the process of field stars subtraction from the on-cluster CMD of NGC 1868. Panel (a) presents the on-cluster CMD resulting from incompleteness correction; panel (b) shows the off-cluster CMD cut by the same guiding lines that we used to define the cluster MS (solid lines). In panel (c) we show the on-cluster stars that were considered as matches to the LMC fields stars in the subtraction 


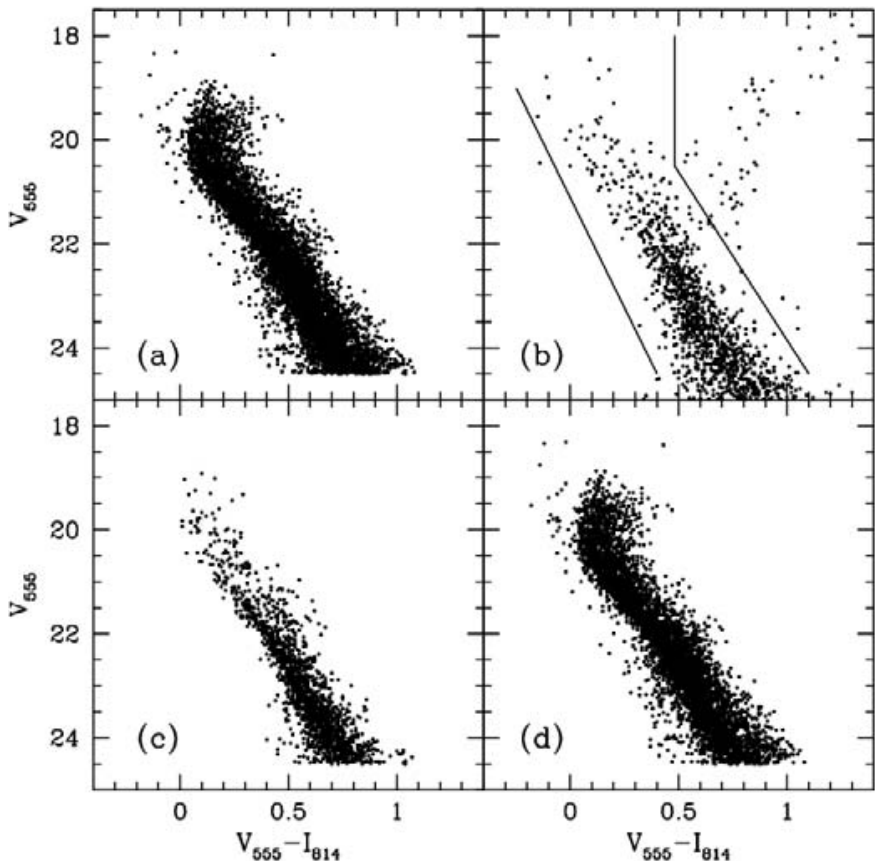

Fig. 7. Correcting the NGC 1868 on-cluster CMD for field star contamination: a) the CMD corrected for sample incompleteness and cut at $\left.V_{555}=24.5 ; \mathbf{b}\right)$ the off-cluster CMD and the solid lines that define the cluster MS; c) the on-cluster stars considered as LMC field stars according to the algorithm described in the text; d) final CMD containing only stars statistically presumed to belong to the cluster.

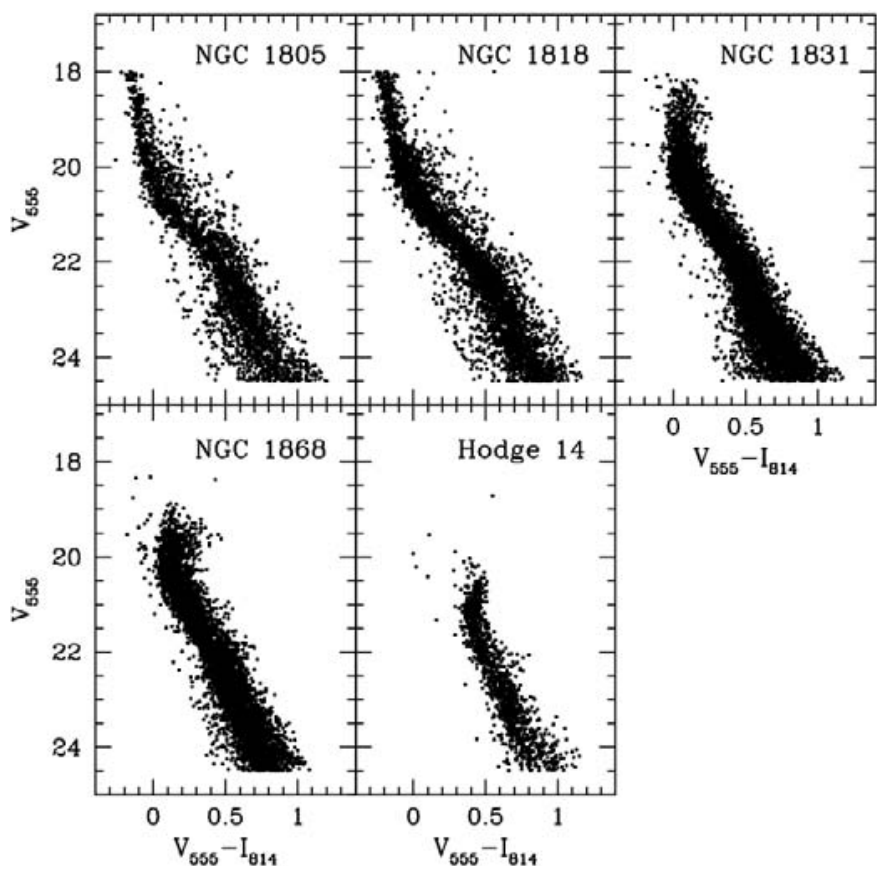

Fig. 8. The final CMDs of all clusters, containing only stars statistically presumed to be cluster members.

process. Finally, the clean and final cluster CMD is shown in panel (d). This final CMD is presented for all clusters in Fig. 8. Comparison with Fig. 3 gives an overall visual idea of the correction results.

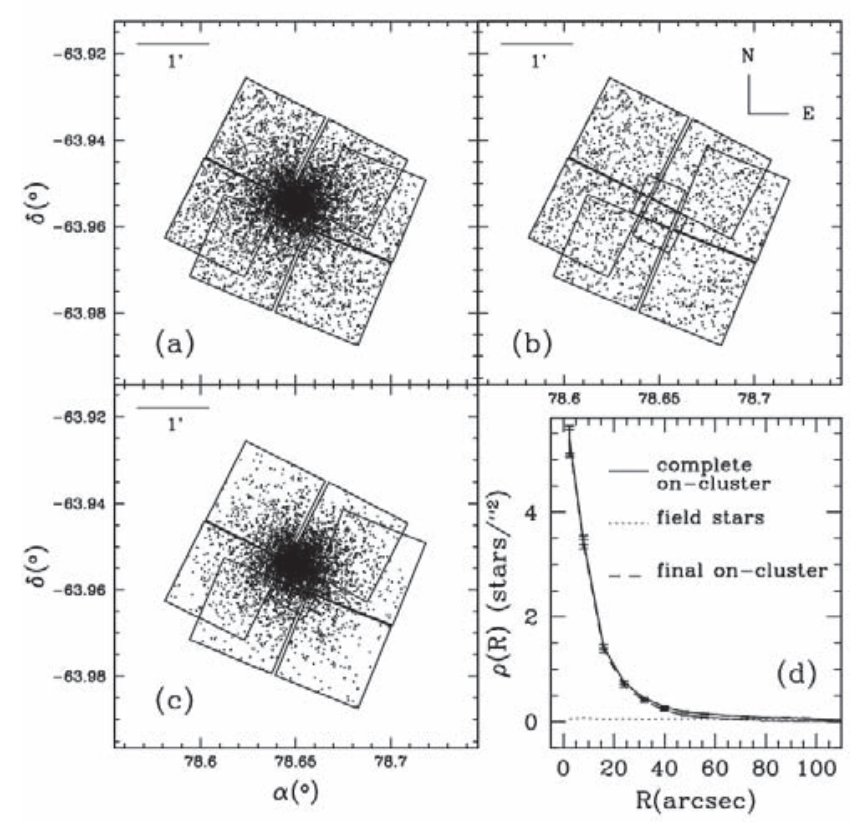

Fig. 9. The sky position of the stars: a) in the complete on-cluster sample; b) statistically found to belong to the field; c) final, field subtracted, cluster sample. The stellar density profiles are shown in panel d).

As this method takes into account the position of stars within the clusters, we expect the density profile of removed field stars to be flat and that of remaining clusters stars to preserve the original shape. This is confirmed in Fig. 9 for NGC 1868, where we show the distributions on the sky of the unsubtracted on-cluster sample (panel (a)), the statistical field stellar sample (panel (b)) and the field subtracted and final cluster sample (panel (c)). Panel (d) shows the evolution of the stellar density profile along the field star decontamination process.

Figure 10 shows, for all clusters, the logarithmic LFs in the $F 555 W$ filter $\left(\Phi\left(V_{555}\right)\right)$ and their variation along the process of correction for the selection effects. The changes in the LFs after each process are quite evident. Completeness correction clearly steepens the original on-cluster LF (solid line), as expected. The field star subtraction process results in a sample whose LF (long-dashed line) is strikingly similar to the off-cluster field LF (short-dashed line). This adds strong confidence to the validity of the decontamination method. The end product of the correction method is an LF (dot-dashed) which is significantly different, and in general shallower, than the original one, emphasizing the importance of these selection effects. Finally, as already seen in the CMDs of Fig. 4, the field LFs differ from each other, attesting to variations in the stellar populations.

Table 4 shows how the number of cluster CMD points evolves during the correction process. Columns 2 and 3 present, respectively, the observed $\left(N_{\text {obs }}\right)$ and incompleteness corrected $\left(N_{\text {comp }}\right)$ number of stars. Column 4 shows the ratio $\left(N_{\text {comp }}-\right.$ $\left.N_{\text {obs }}\right) / N_{\text {obs }}$, indicating that the fractional increase caused by incompleteness correction is always lower than $25 \%$. The number of field stars removed $\left(N_{\text {field }}\right)$ and its fraction relative to $N_{\text {comp }}$ is shown in Cols. 5 and 6 , respectively. Notice that 


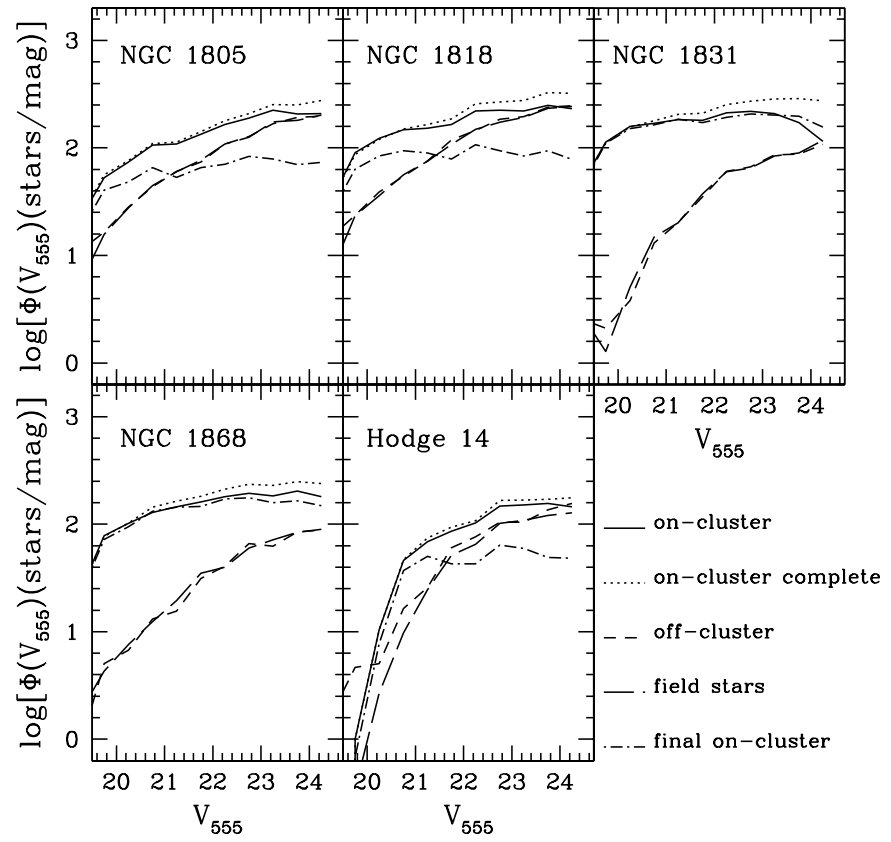

Fig. 10. Evolution of the $V$ band $\operatorname{LF}\left(\Phi\left(V_{555}\right)\right)$ in logarithmic scale for all clusters (as indicated in each panel) along the treatment for selection effects. The line symbols are described on the lower right side of the figure.

Table 4. Number of points in the different stages of correction for selection effects, as described in the text.

\begin{tabular}{lcccccc}
\hline \hline Cluster & $N_{\text {obs }}$ & $N_{\text {comp }}$ & $\frac{N_{\text {comp }}-N_{\text {obs }}}{N_{\text {comp }}}$ & $N_{\text {field }}$ & $\frac{N_{\text {field }}}{N_{\text {comp }}}$ & $N_{\text {clus }}$ \\
\hline NGC 1805 & 5547 & 6350 & 0.13 & 3786 & 0.60 & 2564 \\
NGC 1818 & 7758 & 9395 & 0.17 & 5466 & 0.58 & 3929 \\
NGC 1831 & 7065 & 9094 & 0.22 & 1958 & 0.21 & 7136 \\
NGC 1868 & 6157 & 7360 & 0.16 & 1685 & 0.23 & 5675 \\
Hodge 14 & 2726 & 3033 & 0.10 & 1837 & 0.61 & 1196 \\
\hline
\end{tabular}

NGC 1805, NGC 1818 and Hodge 14 are more contaminated by field stars $(\sim 60 \%)$ than the NGC 1831 and NGC 1868 $(\sim 20 \%)$. These latter are also the richest clusters in our sample, as shown by the final number of cluster stars $\left(N_{\text {clus }}\right)$ in the last column. In all cases, the star counts are restricted to the $18.0 \leq V_{555} \leq 24.5$ range.

\section{CMD modeling}

\subsection{The algorithm}

Using the algorithm described by Kerber et al. (2002) we model the MS of a cluster, in an attempt to reproduce its observational features. The modeling process assumes that the cluster is a single stellar population (SSP) and is graphically described in Fig. 11, and may be separated in the following steps: (a) we fix the metallicity $(Z)$ and age $(\tau)$ of the cluster's SSP by means of a chosen Padova isochrone (Girardi et al. 2000), which defines a sequence of absolute magnitude and colour as a function of mass. We transpose this sequence to the observed CMD plane by means of an intrinsic distance modulus $\left((m-M)_{0}\right)$ and

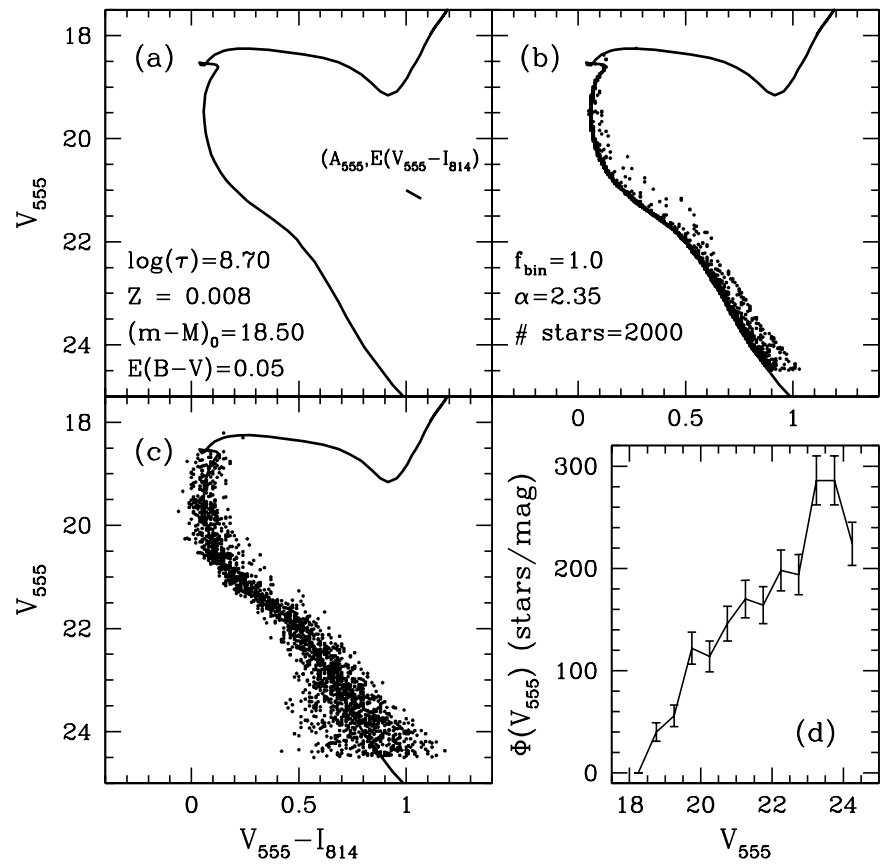

Fig. 11. The CMD modeling process: a) a chosen isochrone with $Z=0.008$ and $\log (\tau / \mathrm{yr})=8.70$, after the introduction of $\left((m-M)_{0}=\right.$ $18.50)$ and $E(B-V)=0.05$; b) 2000 stars randomly drawn from a power-law PDMF, characterized by the slope $\alpha=2.35$. We also apply $f_{\text {bin }}=1.00$, which means that $100 \%$ of the systems are composed of two unresolved stars randomly drawn from the same PDMF; c) the final "observed" version of the model CMD after the introduction of photometric uncertainties; d) the corresponding LF and the $1-\sigma$ Poissonian uncertainties.

reddening $(E(B-V))$; (b) stellar masses are randomly drawn from a power-law PDMF $(\xi(m))$, where the only free parameter is the slope $(\alpha=-\operatorname{dlog} \xi(m) / \operatorname{dlog} m)$. Masses are then converted into absolute magnitudes using the chosen isochrone. We simulate unresolved binarism by randomly drawing an extra stellar mass for a fraction $\left(f_{\text {bin }}\right)$ of cases and combining their fluxes. The number of model systems was equal to the observed number inside the MS region and within the saturation and detection limits. The spread from the isochrone is due to the introduction of a systemic unresolved binarism; (c) we introduce the photometric uncertainties by spreading the stars with Gaussian distributions of errors with $\sigma_{I}$ and $\sigma_{V}$ as empirically determined (see Sect. 2.3); (d) this panel shows the resulting $V$ band LF for the model systems.

Our faintest observed magnitude is $V_{555}=24.5\left(M_{555}=6.0\right.$ for $\left.(m-M)_{0}=18.50\right)$, which corresponds to a lower mass limit of $\sim 0.8 m_{\odot}$. Therefore, our CMD modeling is not sensitive to $\alpha$ in the range $0.08 \leq m / m_{\odot} \leq 0.80$, apart from the effect it has on the modeling of unresolved binaries. We decide to fix $\alpha=1.30$ in this low mass regime. This choice was based in the typical slope found in different stellar populations, as demonstrated by Kroupa (2002). Notice that the range $0.08 \leq m / m_{\odot} \leq 0.15$ is not available in the Padova isochrones, but this has no effect on our CMD modeling for the reasons just stated.

Our choice of system binary fraction, $f_{\text {bin }}$, is a difficult one, as this parameter is unconstrained in rich stellar systems. On the other hand, this parameter was seen to be relatively 
unimportant for our CMD models. The reason is that no a priori mass ratio distribution was assumed, since all masses were drawn from the same unique PDMF. In this way, only about $\sim 20 \%$ of secondary stars end up having large enough flux to cause a significant shift of the system position on the CMD plane relative to the primary. These pairs have a mass ratio $q=\frac{m_{2}}{m_{1}} \gtrsim 0.6$, where $m_{1}$ and $m_{2}$ are the primary and secondary mass, respectively. We thus considered all systems as binaries, making $f_{\text {bin }}=1.00$, with little influence on the results. We plan to fully explore the effect of unresolved binaries on an observed CMD in a future paper.

Therefore we have 5 free model parameters: $Z, \tau,(m-M)_{0}$, $E(B-V)$ and $\alpha$ (for $\left.m>0.80 m_{\odot}\right)$. The 4 first parameters are uniquely defined for each cluster and affect the general shape and position of the MS in the CMD. We call them global parameters. The PDMF slope likely varies as a function of position within the cluster and affects the way stars are spread along the MS. As a consequence, we adopt the following modeling approach:

(1) the global parameters $\left(Z, \log (\tau), E(B-V)\right.$ and $\left.(m-M)_{0}\right)$ are determined using the CMD of the central region imaged by the PC/CEN ( $R \lesssim 20^{\prime \prime}$, where $R$ is the projected distance from the cluster's center). This central region, besides being covered by a single detector, does not suffer from strong field contamination and statistical noise. PDMF slope is fixed $\alpha=2.30$ for $m>0.80 m_{\odot}$ at this stage. The results of this modeling are presented in the this paper.

(2) using the best combination of the global parameters found in step 1 , the position-dependent parameter $\alpha$ is then derived using the LFs or CMDs in concentric rings of variable radii. This is the subject of the following paper.

As mentioned, this strategy naturally splits the parameters into the global and position-dependent ones. We determined the global parameters finding the best models by direct comparison of the observed CMD with artificial ones. The statistical tools used in the model vs. observation comparisons are presented in Sect. 4.

\subsection{The model grids}

The input values for global parameters in the CMD modeling were chosen in order to be consistent with those found in the literature and with the possibilities offered by our data. The web page www . ast . cam.ac.uk/STELLARPOPS/LMCdatabase by Richard de Grijs includes a very large compilation of parameter values and references on the 5 LMC rich clusters analysed in this work.

In accordance with this database and a visual isochrone fit, we set the range of possible values for each physical parameter and created a regular model grid within this range. We expect that use of this systematic grid should prevent biases in the parameter value determination.

\section{$(m-M)_{0}$ and $E(B-V)$}

Values of these parameters found in the literature for all clusters are subject to significant uncertainties since many of

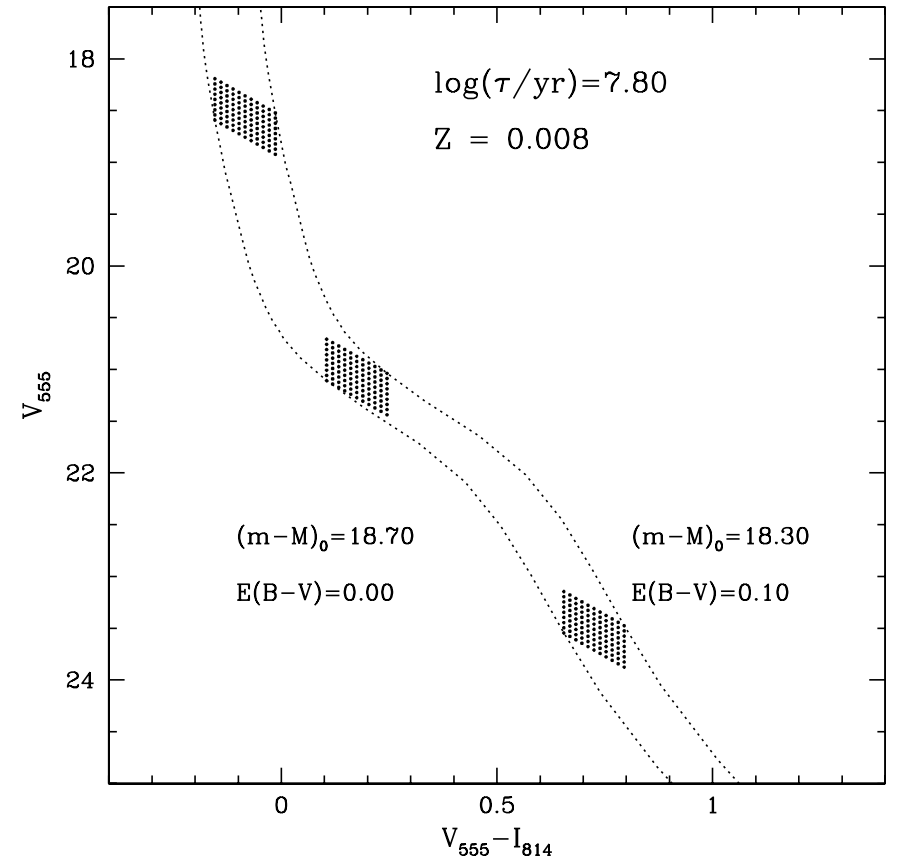

Fig. 12. Effect in the CMD position caused by the allowed range of values for $\left(V-M_{V}\right)_{0}$ and $E(B-V)(18.50 \pm 0.20$ and 0.00 to 0.10 , respectively). The dotted lines limit the extreme positions for the isochrone with $\log (\tau / \mathrm{yr})=7.80$ and $Z=0.008$. Each of the 3 set of points corresponds to the 99 possible CMD positions inside the full explored range.

them are based on visual isochrone fits, which sometimes suffer from a degeneracy of solutions. To avoid any bias imposed by literature results, we explored a wide range of $(m-M)_{0}$ values, as long as they were consistent with the distance to the LMC, its disk size and inclination. Therefore we explored the range $18.30 \leq(m-M)_{0} \leq 18.70$ in steps of 0.05 ; this range is centered at the distance of $\sim 50 \mathrm{kpc}$ found by Panagia et al. (1991) (using purely geometrical arguments applied to high quality imaging and spectral data on supernovae SN1987a), with a spread of $5 \mathrm{kpc}$. Likewise, for $E(B-V)$ we considered a flexible range of values typically found in the LMC direction. The range $0.0 \leq E(B-V) \leq 0.10$ brackets practically all determinations in the LMC direction and was covered in steps of 0.01 . The step sizes were based on the typical photometric sensitivity of our CMD lines.

Figure 12 shows the span in a given CMD position allowed by the range of values for these two parameters ( 99 positions).

\section{$Z$ and $\log (\tau)$}

The range of values of these parameters for each cluster is shown in Table 5, along with the number of model values in each case. The total number of models, which is the product of the number of values listed in the table multiplied by 99 (from the distance modulus and reddening modeling grid) is also given in the last column. Since the stars on the turn-off point of youngest clusters are saturated in our data, we do not determine the age of these systems $\left(n_{\tau}=1\right)$. We thus adopted the youngest Padova isochrone for these clusters, without any further impairment of the CMD modeling process. 
Table 5. Range of metallicity and age used in the modeling process for each cluster. Column 1: cluster name; Col. 2: range of $\log (\tau / \mathrm{yr})$; Col. 3: the total number of age model values; Cols. 4 and 5: the metallicity range and number of model values, respectively; Col. 6: the total number of models.

\begin{tabular}{lccccc}
\hline \hline Cluster & $\log (\tau / \mathrm{yr})$ & $n_{\tau}$ & $10^{2} Z$ & $n_{Z}$ & $n_{\text {tot }}$ \\
\hline NGC 1805 & 7.80 & 1 & $0.2-2.4$ & 8 & 792 \\
NGC 1818 & 7.80 & 1 & $0.1-1.5$ & 7 & 693 \\
NGC 1831 & $8.50-8.80$ & 7 & $0.6-1.9$ & 5 & 3465 \\
NGC 1868 & $8.75-8.95$ & 5 & $0.2-1.5$ & 6 & 2970 \\
Hodge 14 & $9.05-9.35$ & 7 & $0.2-1.5$ & 6 & 4158 \\
\hline
\end{tabular}

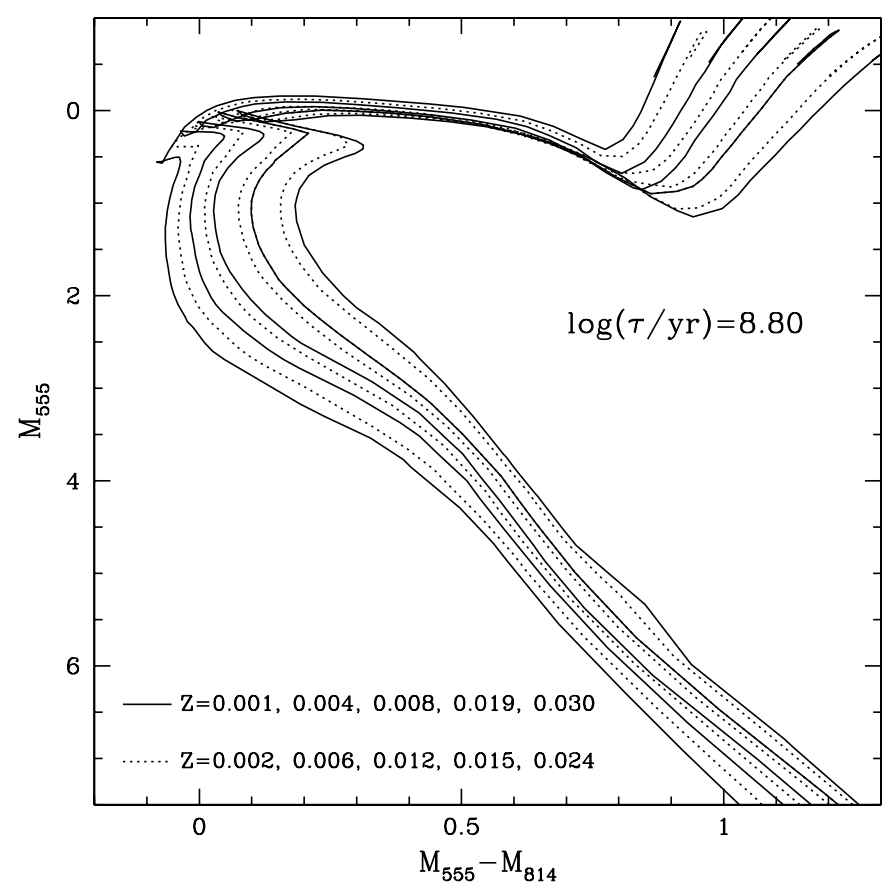

Fig. 13. Isochrones with $\log (\tau / \mathrm{yr})=8.80$ for all available grid. The original Padova grid is shown by solid lines whereas the interpolated one is shown by dotted lines.

The allowed step in age was $\delta \log \tau=0.05$, given by the Padova isochrone original grid. In terms of metallicity, the original grid offered $Z=0.001,0.004,0.008,0.019$ and 0.030 . By means of an isochrone interpolation method in $Z$, we increased this resolution, including new isochrones with $Z=$ $0.002,0.006,0.012,0.015$ and 0.024 . In brief, this method interpolates in mass and magnitude stars that are in equivalent evolutionary phases in two neighboring isochrones of the original grid. Figure 13 shows the original Padova isochrones and the interpolated ones, where it is clear that the interpolation method does not introduce any artificialities in the isochrones.

\section{Statistical tools}

The comparison of data and model CMDs requires objective statistical tools that quantify the agreement between them. The specific statistics used, the criteria to select the best models according to their values and the way the cluster parameter are determined from the best models is variable and depends on the type of analysis carried out. One approach is to replace the two-dimensional distribution of stars along the MS by a fiducial line that delineates its mean place on the CMD plane (see Sect. 2.4.1). This method reduces the CMD comparison to a single dimension, being thus least sensitive to the spread of stars caused by photometric errors and binarism. We call this the $1 \mathrm{D}$ analysis.

\subsection{D analysis}

The fiducial lines were computed in the same way in the model and data CMD, by taking the median colour in different magnitude bins. These lines were used in the data CMDs to correct the sample for incompleteness effects. In order to quantify the proximity of two fiducial lines we use the $\chi^{2}$ statistic defined as follows:

$\chi^{2}=\frac{1}{\left(N_{\mathrm{b}}-1\right)} \sum_{i=1}^{N_{\mathrm{b}}}\left[(V-I)_{\mathrm{obs}, i}-(V-I)_{\bmod , i}\right]^{2}$,

where $(V-I)_{\mathrm{obs}, i}$ and $(V-I)_{\bmod , i}$ are the fiducial line colour $\left(V_{555}-I_{814}\right)$ positions of the $i$ th $V_{555}$ magnitude bin for the observed CMD and model CMD, respectively. $N_{\mathrm{b}}$ is the number of $V_{555}$ magnitude bins used.

This is similar to a colour dispersion between model and observed fiducial lines, therefore being smaller the better a description of the data the model is. As different realizations of a single model may result in different $\chi^{2}$ values, we run 100 realizations of each model and take the mean value and its associated dispersion around the mean. Compatible models for a given observed CMD are then taken as those which satisfy the following criterion

$\chi^{2} \leq \chi_{\min }^{2}+2 \sigma_{\chi^{2}}$,

where $\chi_{\min }^{2}$ is the minimum value averaged over the 100 realizations found among all models, and $\sigma_{\chi^{2}}$ is its associated dispersion. In other words, only models which are within $2 \sigma$ of the best model in the grid are considered compatible descriptions of the data. The mean parameter values among these models then give our best parameter estimates for the cluster; the associated spread among the mean naturally yields the parameter uncertainty.

The whole 1D analysis is depicted in Fig. 14 where panel (a) shows two CMDs being compared and their corresponding fiducial lines. The same lines appear in the next panel, along with their resulting $\chi^{2}$. Finally, the distribution of $\chi^{2}$ over all model realizations and its corresponding average and dispersion values are shown in panel (c).

\section{2. $2 D$ analysis}

Another approach towards defining the models that best describe a given data CMD makes use of the two-dimensional CMD distribution. It clearly explores more CMD information but, as a consequence, is more sensitive to CMD spread, residual field contamination, and other effects. This 2D analysis 


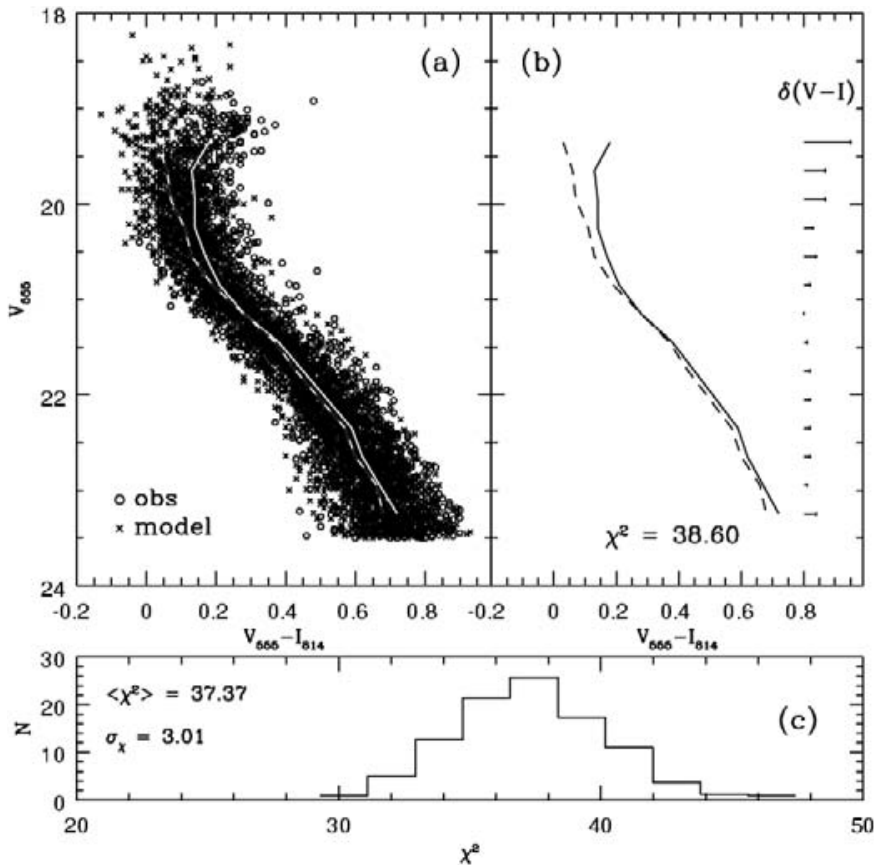

Fig. 14. Panel a): an example of observed and model CMDs compared using the $1 \mathrm{D}$ analysis. The CMDs are shown with different symbols and their fiducial lines are also represented (model CMD: crosses and dashed line; observed CMD: circles and solid line). Panel b): the two fiducial lines are shown again, along with the $(V-I)$ colour difference for each magnitude bin; the resulting value of $\chi^{2}$ is given as well. Panel c): the distribution of $\chi^{2}$ values for 100 realizations of the same model.

requires statistics such as those described in previous papers (Kerber et al. 2001, 2002). We refer the reader to these earlier works for the details on each statistic. We here briefly summarize their properties:

pss: this is an estimate of the joint probability of two point distributions being drawn from the same parent distribution, given their typical fluctuations. It involves ranking the data CMD counts among the model counts in a CMD box grid and finding the percentile deviates of the data counts relative to the median model counts. The larger this difference relative to the model median, the smaller the pss value.

$\chi_{\gamma}^{2}$ : this is similar to a $\chi^{2}$ and has been proposed by Mighell (1999). Kerber et al. $(2001,2002)$ had originally used a simple dispersion $\left(S^{2}\right)$, rather than this more up-to-date one. But essentially this type of statistic computes the dispersion between observed and model counts in a box grid over the CMD.

$\log L$ : this is based on a simple likelihood approach which computes the likelihood of a given data distribution being drawn from a given model. For each model the probability of randomly drawing a star in a given CMD position is calculated, the product of these probabilities over all observed CMD stars being proportional to $L$.

$W$ : proposed originally by Saha (1998) and also used by Valls-Gabaud \& Lastennet (1999) and Lastennet \& Valls-Gabaud (1999), this is also a measure of the probability of data and model being consistent with each other.
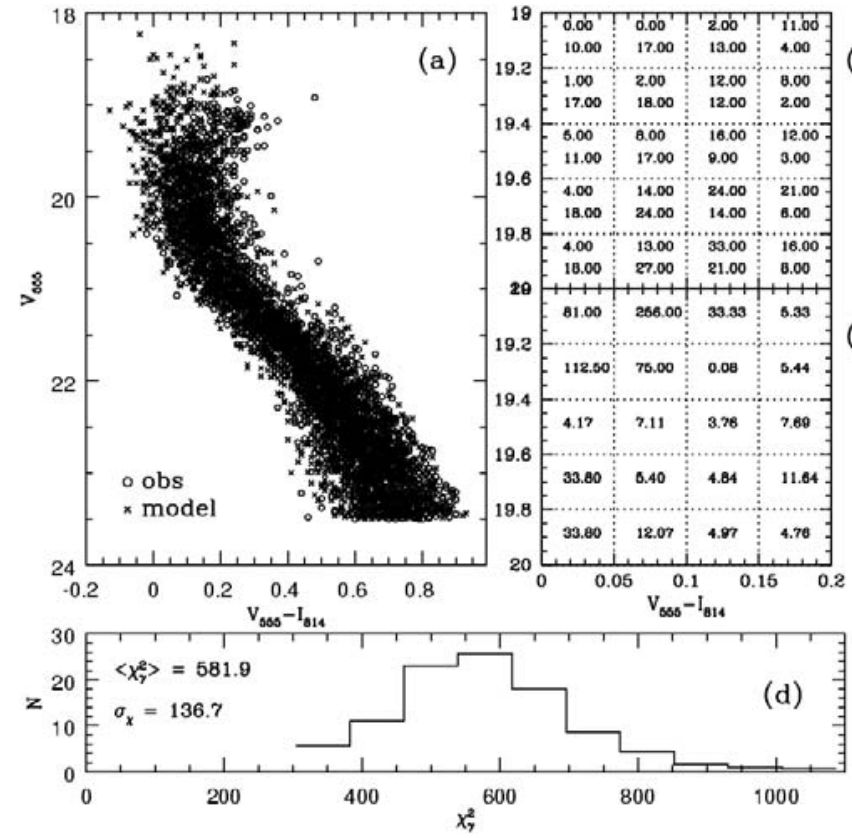

Fig. 15. Panel a): an example of observed and model CMDs compared using the $2 \mathrm{D} \chi_{\gamma}^{2}$. The CMDs are shown with different symbols (model CMD: crosses; observed CMD: circles). Panel b): expansion of the upper CMD region, divided in a box grid. For each box the upper value is the number of stars in the observed CMD, whereas the lower value is the number of model stars shown in panel a). Panel c): the contribution of the same boxes shown in panel b) to the $2 \mathrm{D} \chi_{\gamma}^{2}$. Panel d): the distribution of $\chi_{\gamma}^{2}$ values for 100 model realizations.

Figure 15 is similar to Fig. 14 but now it describes the way the $2 \mathrm{D} \chi_{\gamma}^{2}$ statistic is applied. Notice that different realizations of the same model again yield a distribution of $\chi_{\gamma}^{2}$ values over which an average and dispersion are found and used as criteria in finding the best model for each dataset.

\subsection{Control experiments}

As mentioned earlier, Kerber et al. (2001, 2002) have applied the statistics discussed in the previous section to data CMDs, also built from HST/WFPC2 data. The authors made use of control experiments, by which a fake observation with known input parameters is compared to a model grid in order to assess whether the methods based on model vs. data comparisons is capable of recovering the input parameter values. Here, we briefly discuss these validating tests. In Fig. 16 we show the $\log (W)$ vs. $\log \left(\chi_{\gamma}^{2}\right)$ correlation in the left panels. The panels on the right are a magnification of the regions where the compatible models lie (i.e., the region within $2 \sigma$ of the $\operatorname{largest} \log (W)$ and lowest $\left(\chi_{\gamma}^{2}\right)$ ). The different symbols correspond to models with different metallicities (upper panels) and ages (lower panels) taken from the model grid defined in Sect. 3.2 for NGC 1868. Figure 17 shows similar panels, but now with symbols describing different $\left(V-M_{V}\right)_{0}$ (upper panels) and $E(B-V)$ (lower panels) values.

The input model used to create the fake observed CMD, marked with a large star in all panels, is clearly the one that best resembles the "data", since it yields the most extreme 


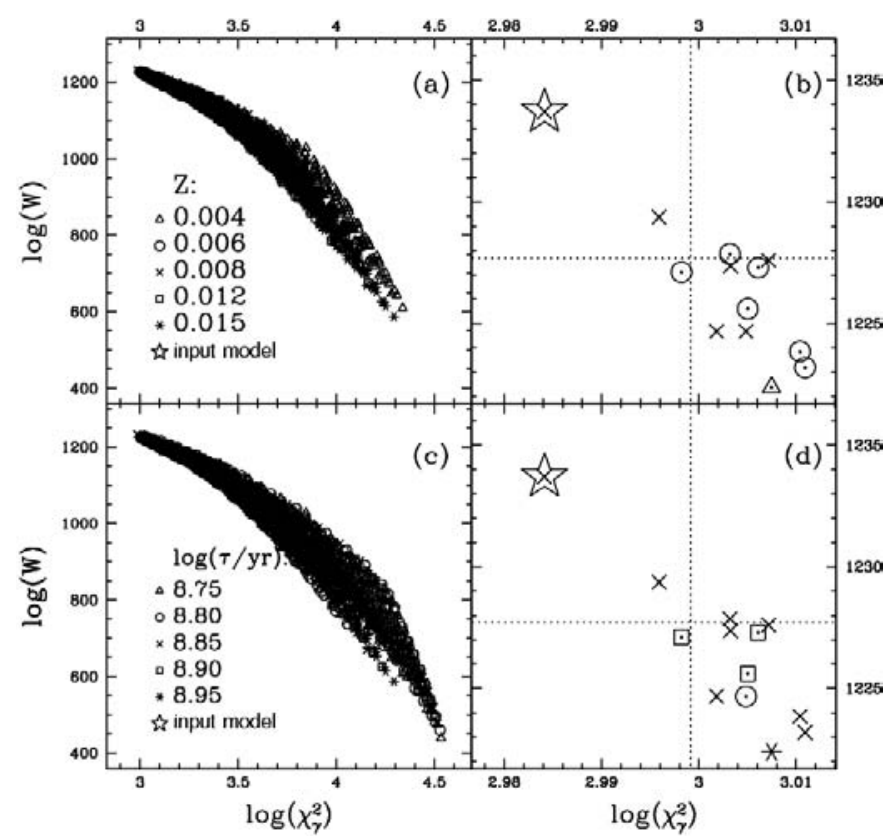

Fig. 16. Panel a): $\log (W)$ vs. $\log \left(\chi_{\gamma}^{2}\right)$ correlation found in a control experiment using the model grid for NGC 1868. The different symbols correspond to the different $Z$ values. Panel b): an expansion of the upper left region of panel a), where the best models are located. The input model, used to create the "observed CMD" used in the data vs. model comparison, is shown as the large star. The dotted lines limit the region where models satisfy a joint $1 \sigma$ criterion on both statistics. Panel c): the same models and statistics as in panel a, but now the symbols identify the age values in the model grid. Panel d): the best models shown again in detail, but now coded according to age.

values of both statistics. The dotted lines in panels (b) and (d) indicate the $1 \sigma$ spread from these extreme values, taken from 100 realizations of the best/input model. Notice that the compatible models shown in these panels have similar parameter values to the input one. Similar conclusions result if we choose other pairs of statistics to compare.

As mentioned in the previous section and illustrated above, one may use the models that satisfy the criterion $\chi^{2} \leq \chi_{\min }^{2}+$ $2 \sigma_{\chi^{2}}$, and similarly the other statistics used in the $2 \mathrm{D}$ analysis, to determine the best model parameter values and their associated uncertainties for a given CMD. In Fig. 18 we show projections of the model grid, in this case the one for Hodge 14, on several parameter planes. The models which satisfy the joint $2 \sigma$ criteria for all four statistics are marked with an $X$; those satisfying the $2 \sigma$ criterion for the 1D analysis are marked with - ; the average values and dispersions obtained with both analyses are also indicated as large crosses.

As this last control experiment makes use of the model grid and CMDs of a much poorer cluster, Hodge 14, the solutions found are not as accurate as in the case of the previous experiment, which faked NGC 1868 data. Therefore, acceptable solutions are more spread out over the parameter grid space, some correlations existing among the parameter values. For example, compatible models with larger ages, which push the turn-off towards redder colours, tend to have lower metallicities, which push the data towards the blue. Hence, this type of diagram reflects in full the limitations of the 1D and 2D CMD analyses

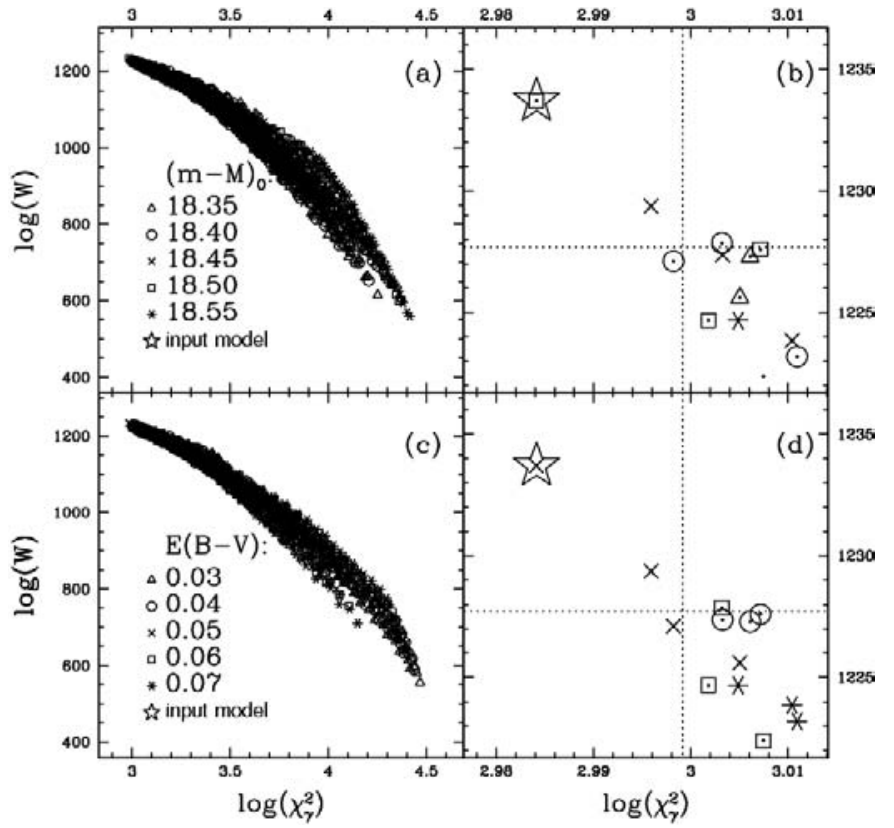

Fig. 17. Panel a): $\log (W)$ vs. $\log \left(\chi_{\gamma}^{2}\right)$ correlation found in a control experiment using the model grid for NGC 1868 . The different symbols correspond to the different $\left(M_{V}-V\right)_{0}$ model values. Panel b): a blow up of the upper left region of panel a), where the best models are located. The input model, used to create the fake "observed CMD" used in the data vs. model comparison, is shown as the large star. Panel c): the same models and statistics as in panel a), but now the symbols identify the $E(B-V)$ values in the model grid. Panel d): the best models shown again in detail, but now coded according to redenning.

Table 6. For each cluster, the global parameters of the input model which generated the fake "observed CMD".

\begin{tabular}{lcccc}
\hline \hline Cluster & $10^{2} Z_{\text {in }}$ & $\log (\tau / \mathrm{yr})_{\text {in }}$ & $(m-M)_{0_{\text {in }}}$ & $E(B-V)_{\text {in }}$ \\
\hline NGC 1805 & 0.80 & 7.80 & 18.50 & 0.050 \\
NGC 1818 & 0.60 & 7.80 & 18.50 & 0.050 \\
NGC 1831 & 1.20 & 8.65 & 18.50 & 0.050 \\
NGC 1868 & 0.80 & 8.85 & 18.50 & 0.050 \\
Hodge 14 & 0.80 & 9.20 & 18.50 & 0.050 \\
\hline
\end{tabular}

and the degeneracies involved therein. It will be used to show the results obtained for the real CMDs of our sample of 5 rich LMC clusters.

As we ran control experiments for the model grids of all 5 clusters, we list their results in the tables that follow. Table 6 lists the input parameters used to generate the CMD that was treated as an "observed CMD". Tables 7 and 8 list the modeling results for the $1 \mathrm{D}$ and $2 \mathrm{D}$ analyses. The recovered parameters are similar in the two methods and are quite close to the input ones, attesting to the validity of both CMD analysis methods. In all cases, the difference between input and output values was within the uncertainties. Also notice that the uncertainties for the experiment based on Hodge 14 tend to be larger than those based on richer clusters, such as NGC 1868 and NGC 1831. 


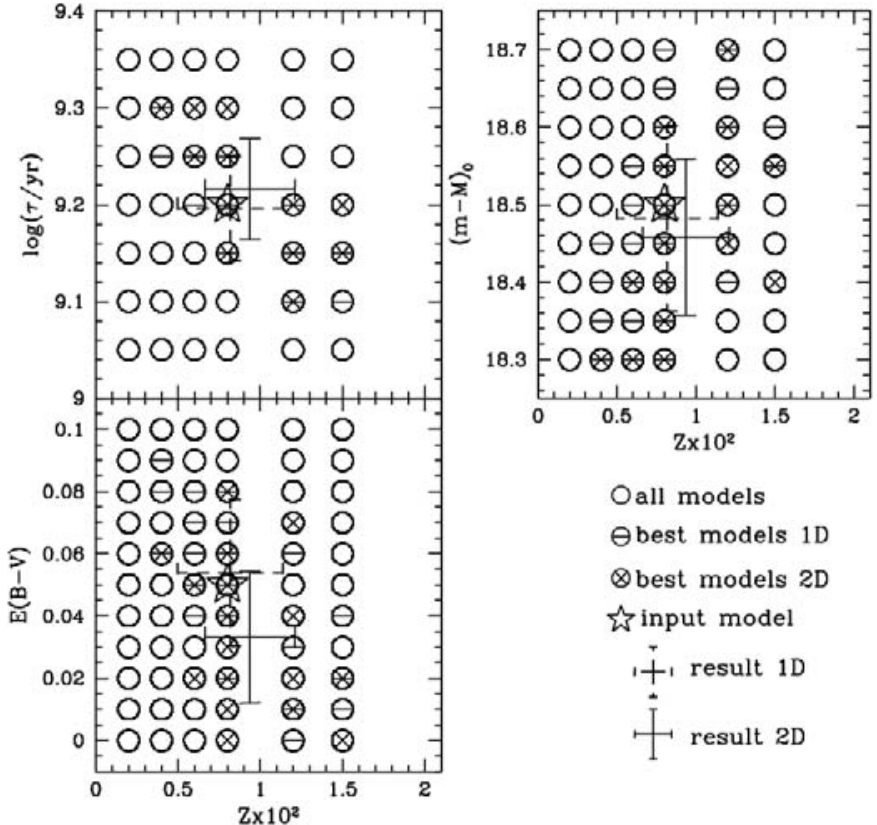

Fig. 18. Panel a): Hodge 14 model grid projected onto the $\log (\tau / \mathrm{yr})$ vs. $Z$ plane. The input model which generated the faked "observed CMD" is shown as a star. Models considered compatible in the 1D analysis are marked with a - , whereas those based on the $2 \mathrm{D}$ analysis are marked with $X$. The average parameter values among these models, and their corresponding uncertainties, are given as a large cross (1D analysis: dashed; 2D analysis: solid). Panel b): the same as in panel a), but now the projection onto the $(m-M)_{0}$ vs. $Z$ plane is shown. Panel c): the same as in panel a), but now showing the projected $E(B-V)$ vs. $Z$ plane.

Table 7. The results of 1D analysis for the control experiments.

\begin{tabular}{lcccc}
\hline \hline Cluster & $10^{2} Z_{\text {out }}$ & $\log (\tau / \mathrm{yr})_{\text {out }}$ & $(m-M)_{0_{\text {out }}}$ & $E(B-V)_{\text {out }}$ \\
\hline NGC 1805 & $0.80 \pm 0.33$ & - & $18.51 \pm 0.13$ & $0.057 \pm 0.009$ \\
NGC 1818 & $0.60 \pm 0.23$ & - & $18.50 \pm 0.12$ & $0.051 \pm 0.010$ \\
NGC 1831 & $1.38 \pm 0.30$ & $8.61 \pm 0.06$ & $18.55 \pm 0.10$ & $0.050 \pm 0.014$ \\
NGC 1868 & $0.75 \pm 0.20$ & $8.88 \pm 0.06$ & $18.47 \pm 0.10$ & $0.050 \pm 0.015$ \\
Hodge 14 & $0.82 \pm 0.32$ & $9.20 \pm 0.05$ & $18.48 \pm 0.12$ & $0.054 \pm 0.024$ \\
\hline
\end{tabular}

Table 8. The results of 2D analysis for the control experiments.

\begin{tabular}{lcccc}
\hline \hline Cluster & $10^{2} Z_{\text {out }}$ & $\log (\tau / \mathrm{yr})_{\text {out }}$ & $(m-M)_{0_{\text {out }}}$ & $E(B-V)_{\text {out }}$ \\
\hline NGC 1805 & $0.81 \pm 0.34$ & - & $18.49 \pm 0.13$ & $0.052 \pm 0.009$ \\
NGC 1818 & $0.58 \pm 0.16$ & - & $18.49 \pm 0.09$ & $0.055 \pm 0.005$ \\
NGC 1831 & $1.36 \pm 0.20$ & $8.63 \pm 0.04$ & $18.54 \pm 0.08$ & $0.051 \pm 0.012$ \\
NGC 1868 & $0.80 \pm 0.10$ & $8.85 \pm 0.03$ & $18.48 \pm 0.03$ & $0.045 \pm 0.005$ \\
Hodge 14 & $0.94 \pm 0.27$ & $9.22 \pm 0.05$ & $18.46 \pm 0.10$ & $0.033 \pm 0.021$ \\
\hline
\end{tabular}

\section{Results}

As mentioned in Sect. 3.1, the global parameters of each cluster were determined using the CMD of the central region imaged by the PC/CEN. These CMDs and their corresponding number of stars are shown in Fig. 19. The model grid was previously

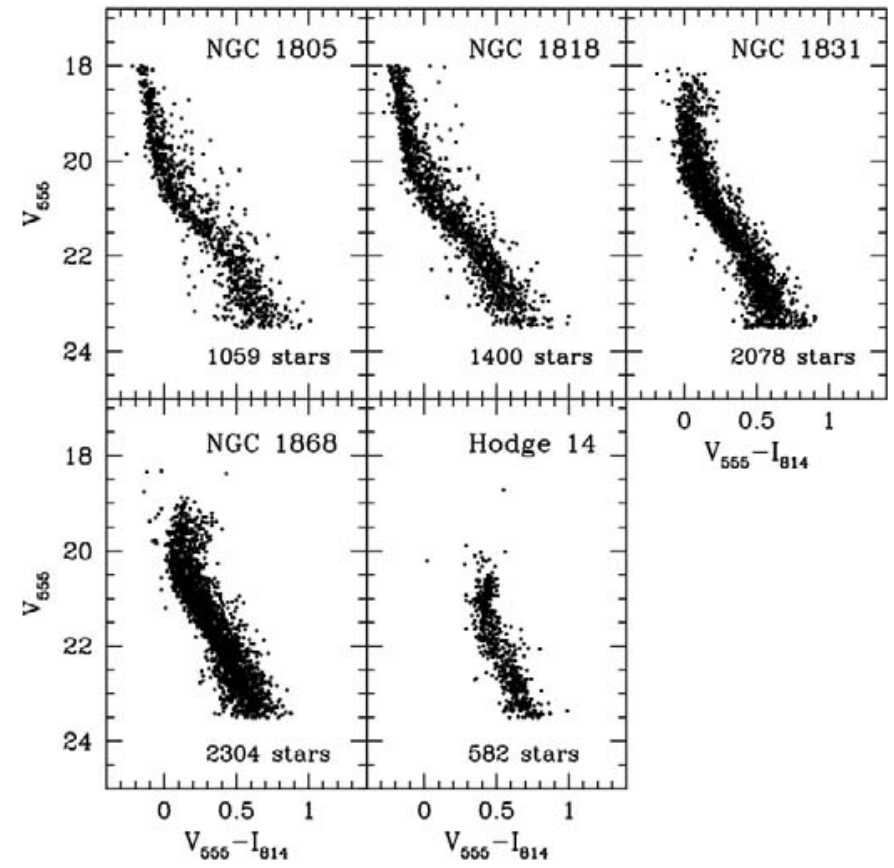

Fig. 19. The final CMDs of all clusters, containing only stars imaged by $\mathrm{PC} / \mathrm{CEN}$.

Table 9. The results of 1D analysis for the central observed CMD.

\begin{tabular}{lcccc}
\hline \hline Cluster & $10^{2} Z$ & $\log (\tau / \mathrm{yr})$ & $(m-M)_{0}$ & $E(B-V)$ \\
\hline NGC 1805 & $0.89 \pm 0.36$ & - & $18.55 \pm 0.11$ & $0.021 \pm 0.012$ \\
NGC 1818 & $0.49 \pm 0.22$ & - & $18.49 \pm 0.14$ & $0.004 \pm 0.006$ \\
NGC 1831 & $1.20 \pm 0.20$ & $8.70 \pm 0.05$ & $18.70 \pm 0.03$ & $0.000_{-0.000}^{+0.005}$ \\
NGC 1868 & $0.68 \pm 0.11$ & $8.93 \pm 0.03$ & $18.63 \pm 0.06$ & $0.004_{-0.004}^{+0.009}$ \\
Hodge 14 & $0.73 \pm 0.33$ & $9.23 \pm 0.07$ & $18.54 \pm 0.13$ & $0.026 \pm 0.023$ \\
\hline
\end{tabular}

presented in Table 5, where we kept $\alpha=2.35$ and $f_{\text {bin }}=1.00$ fixed. Modeling the slope of the PDMF and its positional dependence was left for a separate paper.

In Figs. 20-22 we show the results of $1 \mathrm{D}$ and 2D analyses on the parameter space. Taking into account the uncertainties, in general the results for the two analyses agree very well. Tables 9 and 10 summarise the results for 1D and 2D methods, respectively. As expected by their larger number of stars, the richest clusters (NGC 1831 and NGC 1868) had their parameters better determined than the other clusters. In fact, in a few cases, only a single model satisfied the simultaneous $2 \sigma$ criterion for all statistics adopted. In these cases, we used the bin width along the parameter grid as the uncertainty. As in the case of the control experiments, we again find a correlation between the cluster parameters of the best models, reflecting the degeneracy in the effects they cause on a CMD.

Figure 23 shows the fiducial line representing the observed CMD for the central region of each cluster in comparison with the fiducial line of a CMD realization of the best models. Clearly, the two lines in each panel are very close to each other, indicating that our modeling method successfully recovers the observed CMD positions. Examples of CMD realizations for the best models are presented in Fig. 24. These may be 


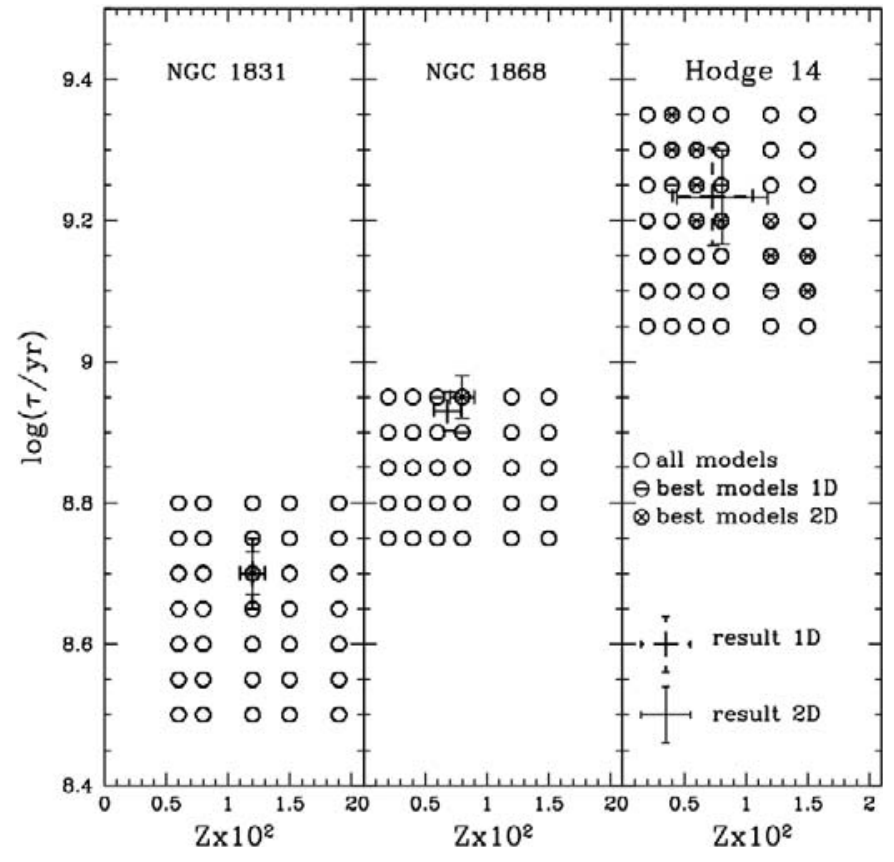

Fig. 20. Results of the $1 \mathrm{D}$ and $2 \mathrm{D}$ analyses on the $\log (\tau)$ vs. $Z$ plane. The symbols are the same as Fig. 18.

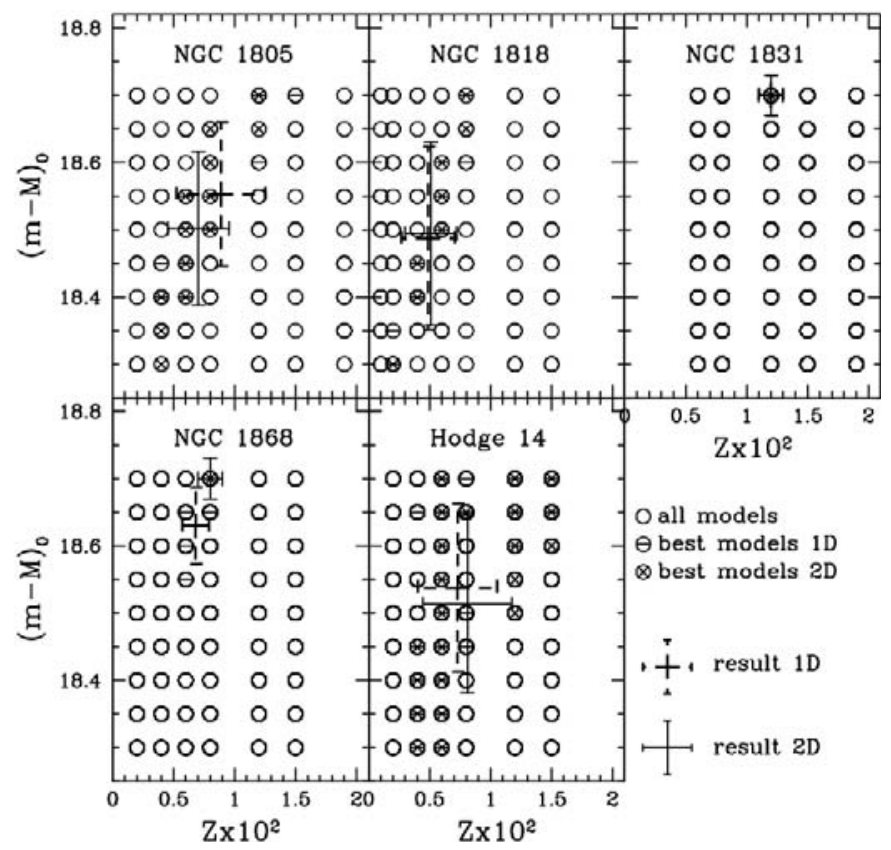

Fig. 21. Results of the $1 \mathrm{D}$ and $2 \mathrm{D}$ analyses on the $(m-M)_{0}$ vs. $Z$ plane. The symbols are the same as Fig. 18.

compared to Fig. 19, where we again confirm the similarity between observed and model CMDs. On the other hand, some secondary observed CMD features have not been reproduced by the models. For the two young clusters, NGC 1805 and NGC 1818, the observed CMD is broader than the model ones. This may be partially due to differential reddening or to the presence of peculiar stars in the upper main sequence (Keller et al. 2000; Johnson et al. 2001). Neither of these effects are incorporated into the models. For NGC 1868, the turn-off region is better defined in the model than in the observed data.

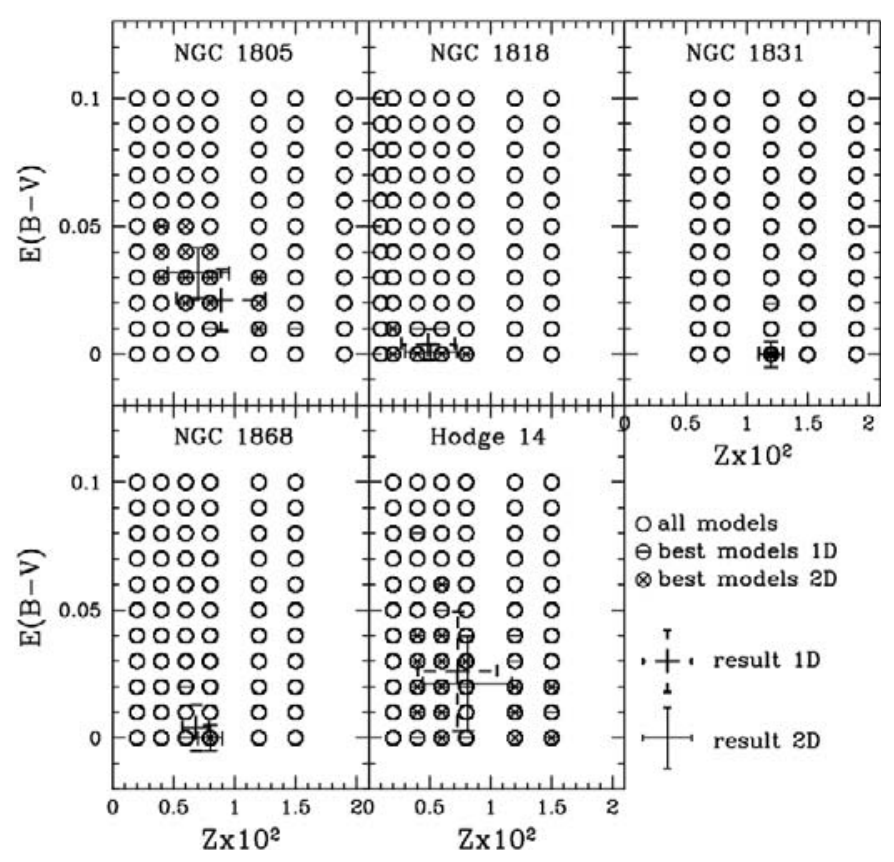

Fig. 22. Results of the $1 \mathrm{D}$ and $2 \mathrm{D}$ analyses on the $E(B-V)$ vs. $Z$ plane. The symbols are the same as Fig. 18.

Table 10. The results of 2D analysis for the central observed CMD.

\begin{tabular}{lcccc}
\hline \hline Cluster & $10^{2} Z$ & $\log (\tau / \mathrm{yr})$ & $(m-M)_{0}$ & $E(B-V)$ \\
\hline NGC 1805 & $0.70 \pm 0.25$ & - & $18.50 \pm 0.11$ & $0.032 \pm 0.010$ \\
NGC 1818 & $0.51 \pm 0.21$ & - & $18.49 \pm 0.14$ & $0.001_{-0.000}^{+0.003}$ \\
NGC 1831 & $1.20 \pm 0.20$ & $8.70 \pm 0.03$ & $18.70 \pm 0.03$ & $0.000_{-0.000}^{+0.005}$ \\
NGC 1868 & $0.8 \pm 0.15$ & $8.95 \pm 0.03$ & $18.70 \pm 0.03$ & $0.000_{-0.000}^{+0.005}$ \\
Hodge 14 & $0.81 \pm 0.37$ & $9.23 \pm 0.07$ & $18.51 \pm 0.13$ & $0.021 \pm 0.019$ \\
\hline
\end{tabular}

Below we give a brief summary of the comparison of our results with others works. In general, there is a considerable discrepancy among the results found in the literature, which can be credited to the uncertainties of each method of analysis and to the great difference in quality between the ground based and the HST data.

We stress that our results are based in statistical models vs. data comparisons that explored a regular grid in 4 dimensional parameter space. This approach prevents biases in the determination of parameter values and objectively reveals the possible degeneracies present in such determinations.

\section{NGC 1805}

The determination of $E(B-V)$ using ground based data is in the range between 0.07 and 0.10 (Persson et al. 1983) (IR photometry); Meurer et al. 1990 (integrated IUE colour). However, HST data for nearby fields (Castro et al. 2001; Javiel et al. 2005) and the extinction maps from Burstein \& Heiles (1982) (hearafter BH82) all suggest smaller values: $0.03 \leq$ $E(B-V) \leq 0.04$. Our results of $E(B-V)$ confirm this tendency. Metallicity values span from $[\mathrm{Fe} / \mathrm{H}]=-0.30$ (Meliani et al. 1994 ) to $[\mathrm{Fe} / \mathrm{H}]=0.0$ (Johnson et al. 2001, who use isochrone fits to HST data). Our inferred metallicity $([\mathrm{Fe} / \mathrm{H}] \approx-0.4)$ is closer to Meliani et al. (1994); however, notice that the optical 


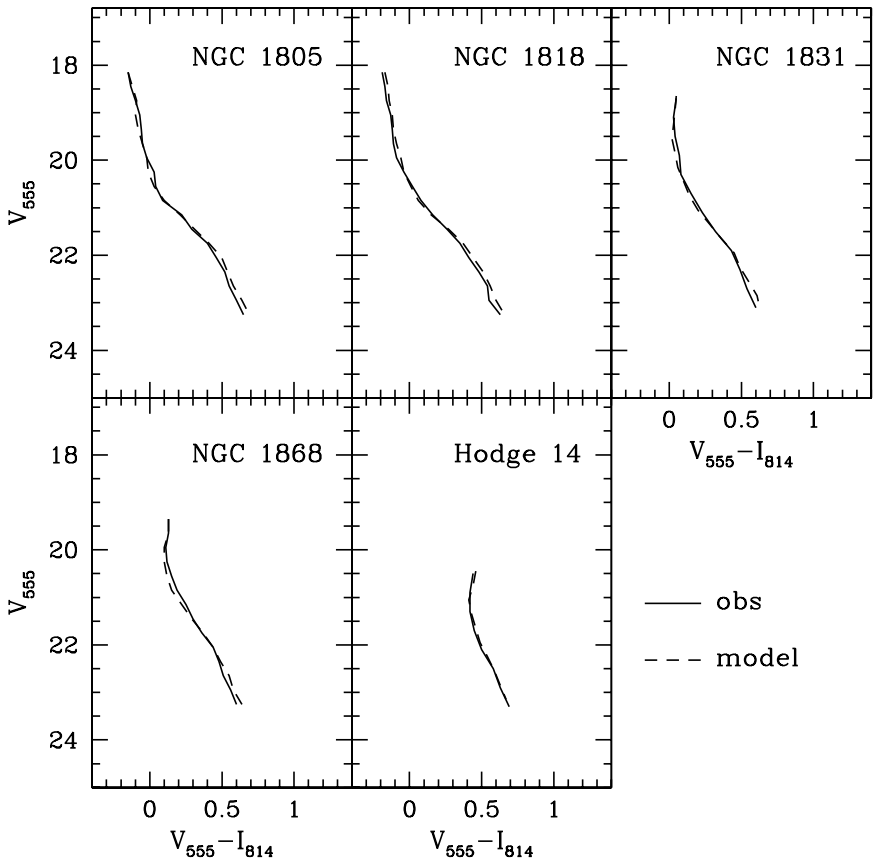

Fig. 23. The fiducial lines for the PC/CEN stars in comparison with examples of fiducial lines for the best models.

CMD shown by Johnson et al. (2001) is also consistent with $[\mathrm{Fe} / \mathrm{H}] \approx-0.4$, especially in the upper MS region.

\section{NGC 1818}

$E(B-V)$ values for this cluster are similar to NGC 1818: ground based data indicate $0.05 \leq E(B-V) \leq 0.10$ (Persson et al. 1983; Cassatella et al. 1987 (IUE spectroscopy); Meurer et al. 1990; Grebel 1997 (isochrone fit)), while HST data and the extinction map from BH82 suggest $0.03 \leq E(B-V) \leq 0.05$ (Hunter et al. 1997; Castro et al. 2001; Javiel et al. 2005).

For metallicity, the most reliable determinations are within $-0.9 \lesssim[\mathrm{Fe} / \mathrm{H}] \lesssim-0.4$ (Meliani et al. 1994 (spectra of 5 red supergiants); Bonatto et al. 1995 (UV spectra); Oliva \& Origlia 1998 (IR spectra)). Our result $([\mathrm{Fe} / \mathrm{H}] \approx-0.6)$ is consistent with this range. On the other hand, isochrone fits for HST data made by Johnson et al. (2001) reached solar metallicity, although their fit using $[\mathrm{Fe} / \mathrm{H}] \lesssim-0.4$ is also adequate for their data.

\section{NGC 1831}

Ground-based $E(B-V)$ values again tend to be larger than HST ones. Mateo (1988) finds $E(B-V)=0.04$ with optical isochrone fits, while Persson et al. (1983) obtain $E(B-V)=$ 0.10 based on IR photometry. Our value of $E(B-V)=0.00$ is similar to those by Castro et al. (2001) and Javiel et al. (2005), both based on HST optical photometry of field LMC stars. Such a small extinction is also quoted in the BH82 maps as well.

For metallicities, a wide range of $-1.0 \leq[\mathrm{Fe} / \mathrm{H}] \leq 0.0$ is found in the literature. Our value $([\mathrm{Fe} / \mathrm{H}]=-0.2)$ is closer to the upper boundary of these estimates, where most of the more recent determinations are also found (Olszewski et al. 1991; Vallenari et al. 1992; Bonatto et al. 1995). Ages also span a significant range in the literature. More recent determinations are within $8.4 \leq \log (\tau / \mathrm{yr}) \leq 8.7$ (Meurer et al. 1990; Olszewski et al. 1991; Vallenari et al. 1992; Bonatto et al. 1995;

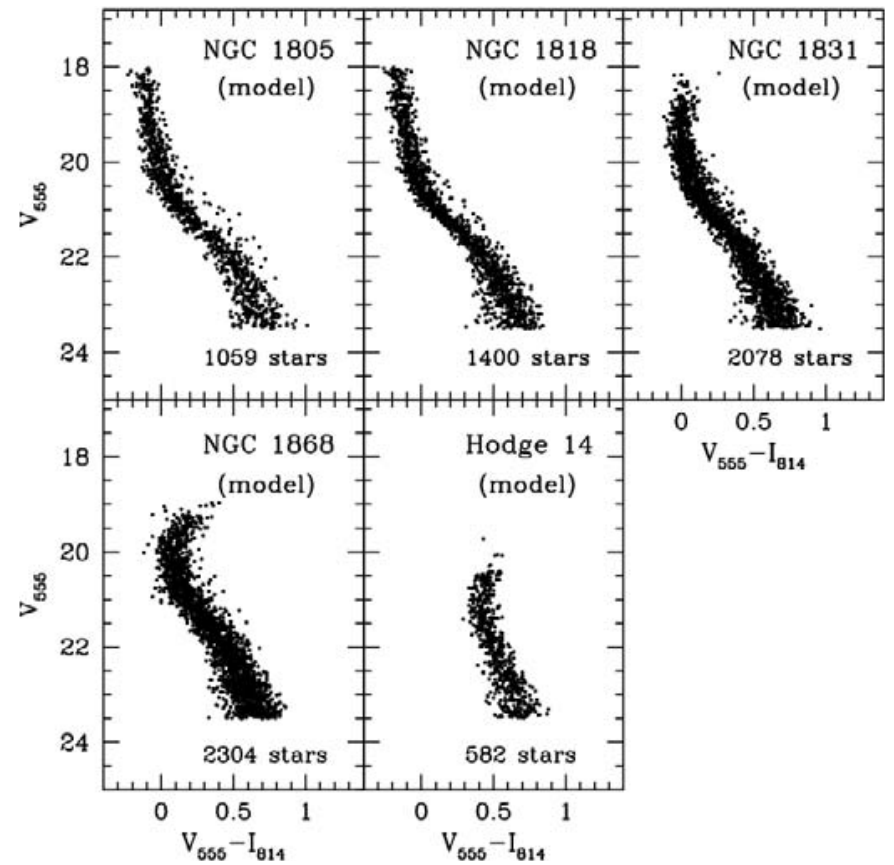

Fig. 24. Examples of best model CMDs according both statistical analysis.

Girardi et al. 1995). In this paper we find $\log (\tau / \mathrm{yr})=8.70 \pm$ 0.03 . We note that the Kerber et al. (2002) preliminary analysis of this cluster yielded a $\operatorname{larger} \log (\tau / \mathrm{yr})$ by $0.05-0.10$, which was caused by the presence of saturated stars in their CMD which mimicked an older turn-off. These stars have been eliminated from the present work.

\section{NGC 1868}

Once more, $E(B-V)$ follows the same behavior described previously: a larger value of $E(B-V)=0.07$ from groundbased data (Persson et al. 1983; Meurer et al. 1990), while $E(B-V)=0.01$ from HST data (Castro et al. 2001) and from the BH82 maps; this lower reddening is again confirmed in the present work. Metallicity values from the literature indicate $[\mathrm{Fe} / \mathrm{H}] \leq-0.50$ (Chiosi et al. 1986; Olszewski et al. 1991), which is marginally consistent with our best determination $([\mathrm{Fe} / \mathrm{H}]=\approx-0.4 \pm 0.1)$. As for ages, Olszewski et al. (1991) and Girardi et al. (1995) constrain the value for this parameter to the range $8.70 \leq \log (\tau / \mathrm{yr}) \leq 9.00$, while our best model is near the upper limit.

\section{Hodge 14}

The same tendency of smaller values of $E(B-V)$ based on HST data is again noticed. However, in this case the value of BH82 $(E(B-V)=0.11)$ is closer to that obtained using groundbased data $(E(B-V)=0.09$, Bomans et al. 1995) than to the value of $E(B-V)=0.04$ obtained by Castro et al. (2001) based on HST data. Our result is consistent with this latter. In terms of metallicity, our recovered value of $([\mathrm{Fe} / \mathrm{H}] \approx-0.4 \pm 0.2)$ is consistent with the upper limit found in the literature $([\mathrm{Fe} / \mathrm{H}]=$ $-0.66 \pm 0.2$ ) (Jensen et al. 1988; Olszewski et al. 1991). As for ages, the more recent works restrict to this parameter to the range $9.15 \leq \log (\tau /$ yr $) \leq 9.40$ (Olszewski et al. 1991; Bomans et al. 1995; Geisler et al. 1997); this is also where our best solution lies. 
Thus, apart from systematically smaller extinction values relative to those based on ground observations, the parameters of our best models tend to agree with several estimates found in the literature, especially in more recent works, our error bars being in general smaller. As for $\left(V-M_{V}\right)_{0}$, most authors use a priori values for this parameter, rather than leaving it free to vary. The uncertainties we find here for the distance moduli are $\lessgtr 0.15$, again providing stronger constraints to this parameter than previously obtained.

\section{Summary and conclusions}

In this work we analysed deep CMDs of five rich LMC clusters (NGC 1805, NGC 1818, NGC 1831, NGC 1868 and Hodge 14) obtained from HST/WFPC2 images in the $F 555 \mathrm{~W}$ and $F 814 W$ filters. Comparing the observed CMDs, corrected for selection effects, with model CMDs for a single population, we inferred the metallicity, the intrinsic distance modulus and the reddening for all clusters. We also determined the age for the three oldest clusters (NGC 1831, NGC 1868 and Hodge 14), whose turn-offs were available in our CMDs.

To correct the observed CMDs for sample incompleteness and field star contamination we developed and applied statistical methods. Incompleteness corrections made use of extensive simulations of artificial stars and take into account completeness variations with position within the cluster. Correction for field star contamination was based on CMD comparisons with nearby off-cluster fields and has resulted in a sample of subtracted stars uniformly distributed on the sky and with a luminosity function strongly resembling that of real field stars. The model CMDs were developed in order to reproduce the observational features of the observed one, such as the spread in colour due to unresolved binarism and to photometric uncertainties. The data vs. model comparison was made by means of statistical techniques in 1D and 2D, allowing us to discriminate objectively the models that best reproduce the data. These statistical techniques were tested by means of realistic control experiments, in which a model CMD with known input parameters was used as "the observed data". The experiments proved that the method successfully recovers the input model in all parameter grids adopted. We used regular model grids in the parameter space to prevent possible biases in the determination of the best solutions.

Tables 9 and 10 summarize the results in 1D and 2D analysis, respectively. Taking into account the uncertainties, these results are in accordance, adding reliability to our determined physical parameters. A number of best models is found for each cluster, which reveals a degeneracy in the parameter values. In fact, different choices of extinction, distance modulus, metallicity and age may result in isochrones occupying nearly exactly the same locus in a CMD. This effect by itself accounts for such a degeneracy in parameter space. On the other hand, for rich enough clusters, such as NGC 1868 and NGC 1831, our numerical methods, which make intense use of all CMD information available, raise this degeneracy to a large extent. We also stress that, in general, the results found here have uncertainties much smaller than the range covered by the model grid and the range of solutions found in the literature.
In an upcoming paper we will use these results for the global parameters to determine the positional dependence of the PDMF slope, thus quantifying the effect of mass segregation and stellar evaporation in the clusters. As mentioned in the Introduction, modeling of the PDMF and of the current structure of a cluster is an important step towards recovering the initial conditions at the time it formed and assessing, among other things, the universality of the IMF and whether mass segregation takes place at formation or only after dynamical evolution.

Finally, the CMD modeling and comparison methods developed in this and in our earlier works may be applied to a wide range of other photometric data, such as cluster or field CMDs of Galactic populations or of more distant galaxies, as larger and better resolution instruments become available.

Acknowledgements. We thank David Valls-Gabaud for the earlier version of several of our modeling codes. We also thank Carme Gallart and Sandro Javiel for useful discussions. We acknowledge CNPq and PRONEX/FINEP 76.97.1003.00 for partially supporting this work.

\section{References}

Beaulieu, S., Elson, R., Gilmore, G., et al. 1999, New Views of the Magellanic Clouds, ed. Y.-H. Chu, N. Suntzeff, J. Hesser, \& D. Bohlender, IAU Symp., 190, 460

Bica, E., Geisler, D., Dottori, H., et al. 1998, AJ, 116, 723

Bomans, D. J., Vallenari, A., \& de Boer, K. S. 1995, A\&A, 298, 427

Bonatto, C., Bica, E., \& Alloin, D. 1995, A\&AS, 112, 71

Brocato, E., Di Carlo, E., \& Menna, G. 2001, A\&A, 374, 523

Burstein, D., \& Heileis, C. 1982, AJ, 87, 1165 (BH82)

Carrera, R., Aparício, A., Martínez-Delgado, D., \& Alonso-Garcia, J. 2002, AJ, 123, 3199

Casertano, S., \& Muchtler, M. 1998, WFPC2 Instrument Science Report 98-02

Cassatella, A., Barbero, J., \& Geyer, E. H. 1987, ApJS, 64, 83

Castro, R., Santiago, B., Gilmore, G., Beaulieu, S., \& Johnson, R. 2001, MNRAS, 326, 333

Chiosi, C., Bertelli, G., Bressan, A., \& Nasi, E. 1986, A\&A, 165, 84

Chiosi, C. 1989, RMxAA, 18, 125

de Grijs, R., Johnson, R., Gilmore, G., \& Frayn, C. 2002a, MNRAS, 331,228

de Grijs, R., Gilmore, G., Johnson, R., \& Mackey, A. 2002b, MNRAS, 331,245

de Grijs, R., Gilmore, G., Mackey, A., et al. 2002c, MNRAS, 337, 597

Dolphin, A. E. 2002, MNRAS, 332, 91

Elson, R., Gilmore, G., Gerard, F., \& Santiago, B. 1997, MNRAS, 289,157

Elson, R., Tanvir, N., Gilmore, G., Johnson, R., \& Beaulieu, S. 1999, New Views of the Magellanic Clouds, ed. Y.-H. Chu, N. Suntzeff, J. Hesser, \& D. Bohlender, IAU Symp., 190, 417

Gallart, C., Freedman, W. L., Aparício, A., Bertelli, G., \& Chiosi, C. 1999, AJ, 118, 2245

Geisler, D., Bica, E., Dottori, et al. 1997, AJ, 114, 1920

Girardi, L., Chiosi, C., Bertelli, G., \& Bressan, A. 1995, A\&A, 298, 87

Girardi, L., Bressan, A., Bertelli, G., \& Chiosi, C. 2000, A\&AS, 141, 371

Goodwin, S. P. 1997, MNRAS, 286, 669

Grebel, E. K. 1997, A\&A, 317, 448

Hernandez, X., Valls-Gabaud, D., \& Gilmore, G. 1999, MNRAS, 304, 705 
Hernandez, X., Gilmore, G., \& Valls-Gabaud, D. 2000, MNRAS, 317, 831

Hunter, D., O’Neil, E., Lynds, R., et al. 1996, ApJ, 459, L27

Hunter, D., Light, R., Holtzman, J., et al. 1997, AJ, 478, 124

Javiel, S., Santiago, B., \& Kerber, L. 2005, A\&A, 431, 73

Jensen, J., Mould, J. R., \& Reid, I. N. 1988, ApJS, 67, 77

Johnson, J., Bolte, M., Stetson, P., Hesser, J., \& Sommerville, R. 1999, ApJ, 527, 199

Johnson, R., Beaulieu, S., Gilmore, G., et al. 2001, MNRAS, 324, 367

Keller, S., Bessel, M., \& Da Costa, G. 2000, AJ, 119, 1748

Kerber, L., Javiel, S., \& Santiago, B. 2001, A\&A, 365, 424

Kerber, L., Santiago, B., Castro, R., \& Valls-Gabaud, D. 2002, A\&A, 390,121

Kroupa, P. 2002, Science, 295, 88

Kroupa, P., Aarseth, S., \& Hurley, J. 2001, MNRAS, 321, 699

Lastennet, E., \& Valls-Gabaud, D. 1999, RMxA Conf. Ser., 8, 115

Mackey, A., \& Gilmore, G. 2003, MNRAS, 338, 85

Mateo, M. 1988, ApJ, 331, 261

Meliani, M. T., Barbuy, B., \& Richtler, T. 1994, A\&A, 290, 753

Meurer, G. R., Cacciari, C., \& Freeman, K. C. 1990, AJ, 99, 1124
Mighell, K. J. 1999, ApJ, 518, 380

Oliva, E., \& Origlia, L. 1998, A\&A, 332, 46

Olsen, K., Hodge, P., Mateo, M., et al. 1998, MNRAS, 300, 665

Olszewski, E. W., Schommer, R. A., Suntzeff, N. B., \& Harris, H. C. 1991, AJ, 101, 515

Olszewski, E., Suntzeff, N., \& Mateo, M. 1996, ARA\&A, 34, 511

Panagia, N., Gilmozzi, R., Macchetto, F., Adorf, H.-M., \& Kirshner, R. 1991, ApJ, 380, L23

Paresce, F., \& de Marchi, G. 2000, ApJ, 534, 870

Persson, S. E., Aaronson, M., Cohen, J. G., Frogel, J. A., \& Matthews, K. 1983, ApJ, 266, 105

Saha, P. 1998, AJ, 115, 1206

Santiago, B., Beaulieu, S., Johnson, R., \& Gilmore, G. 2001, A\&A, 369, 74

Sarajedini, A., Geisler, D., Harding, P., \& Schommer, R. 1998, ApJ, 508, L37

Valls-Gabaud, D., \& Lastennet, E. 1999, RMxA Conf. Ser., 8, 111

Vallenari, A., Chiosi, C., Bertelli, G., Meylan, G., \& Ortolani, S. 1992, AJ, 104, 1100

Westerlund, B. E. 1990, A\&ARv, 2, 29 